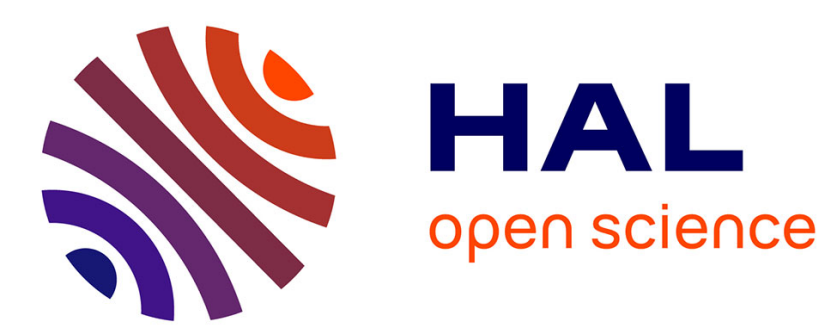

\title{
Du couteau au boucher: remarques préliminaires sur la préparation et le commerce de la viande à Pompéi
}

Nicolas Monteix

\section{To cite this version:}

Nicolas Monteix. Du couteau au boucher : remarques préliminaires sur la préparation et le commerce de la viande à Pompéi. Food and History, 2007, 5 (1), pp.169-195. halshs-00618971

\section{HAL Id: halshs-00618971 \\ https://shs.hal.science/halshs-00618971}

Submitted on 4 Sep 2011

HAL is a multi-disciplinary open access archive for the deposit and dissemination of scientific research documents, whether they are published or not. The documents may come from teaching and research institutions in France or abroad, or from public or private research centers.
L'archive ouverte pluridisciplinaire HAL, est destinée au dépôt et à la diffusion de documents scientifiques de niveau recherche, publiés ou non, émanant des établissements d'enseignement et de recherche français ou étrangers, des laboratoires publics ou privés. 


\title{
Du couteau au boucher : remarques préliminaires sur la préparation et le commerce de la viande à Pompéi
}

\author{
Nicolas Monteix*
}

\begin{abstract}
Butchery is attested in Pompeii only through the presence of the macellum, the public building in which meat was most likely carved and sold. More information is given by inscriptions and frescoes which allow us to gain a more specific idea of the meat consumed. The excavations started in 1748 have revealed the presence of numerous knifes. Some of these have been destroyed during the allied bombing of the city in 1943 or data recording their recovery and original contexts have been lost before they were put away in repositories. As a result only a few are currently studied in this article. By comparing some of these knives with those depicted on funerary monuments, we managed to come with a classification of the tools used in butchery : two kinds of cleaver, long knife and small triangular blade knife. The contextualization of these knives allows us to suggest a new image of the meat market in Pompeii, in which several actors depended on the butcher's craft [169].
\end{abstract}

Les activités de boucherie ne sont directement attestées à Pompéi que par la présence du macellum, édifice public dans lequel la viande était probablement débitée et vendue. Toutefois, des graffites et des fresques permettent d'avoir une idée plus précise de la nature de la viande consommée. Parallèlement, de nombreux couteaux ont été découverts lors des fouilles depuis 1748. Si certains d'entre eux ont disparu, détruits lors du bombardement allié de 1943, ou ont perdu leur contexte de découverte lors de leur transfert dans les dépôts, une partie d'entre eux est analysée dans cet article. En dressant une comparaison entre ces couteaux et ceux qui sont représentés sur des stèles funéraires, il est possible de définir quels étaient les instruments utilisés dans le cadre des activités bouchères : couperet, couteau long, feuille et petit couteau à lame triangulaire. La remise en contexte de ces couteaux autorise à proposer une vision nouvelle du marché de la viande à Pompéi, comportant plusieurs acteurs dépendant toutefois du savoir-faire des bouchers.

En dépit de l'image parfois idyllique véhiculée par Pompéi, l'étude des pratiques de boucherie dans cette ville ne peut se faire que selon des principes inverses à ceux qui président les recherches sur ce sujet dans n'importe quel autre site : l'absence de dépotoir de boucherie identifié, ou tout du moins dont les éléments auraient été conservés ou publiés, empêche d'étudier les traces de découpe sur les os. La seule possibilité pour percevoir les boucheries consiste à caractériser les instruments utilisés pour le débitage de la viande, soit les couteaux. Toutefois, leur identification et leur localisation posent de

* Outre W. van Andringa, qui m'a suggéré de réaliser cette étude, je voudrais remercier P.G. Guzzo, Soprintendente archeologo di Pompei, ainsi qu'A. d'Ambrosio, directeur du site, pour m'avoir autorisé à effectuer cette recherche. L'étude des couteaux conservés dans les dépôts de Pompéi n'aurait guère été possible sans la gentillesse et la disponibilité de G. Cesarano et de G. Gallo. nombreux problèmes, majoritairement liés à l'histoire des fouilles depuis 1748. À cause de ceux-ci, les résultats qui seront ici proposés ne sauraient être que provisoires.

\section{Les apports de l'archéozoologie}

Avant d'aborder ces difficultés propres à Pompéi, il convient de dresser un rapide bilan des apports essentiels de l'archéozoologie à l'étude des pratiques de boucherie. Tout d'abord, je ne mentionnerai pas les questions [170], d'ordre essentiellement écono-
Qu'ils en soient vivement remerciés. Les photos des fresques et des couteaux de Pompéi (Fig. $10 ; 12 ; 13$; 15, a ; 16, b-c ; 17 ; 19) ont été réalisées, sauf mention contraire, par l'auteur, sur concession du Ministero per i Beni e le Attività Culturali - Soprintendenza Archeologica di Pompei. Toute reproduction, par quelque moyen que ce soit, reste interdite. 
mique, liées à l'âge d'abattage : le faible nombre de dépôts mis au jour, étudiés ou conservés à Pompéi empêche de soulever de tels problèmes. En ce qui concerne les espèces consommées, comme nous le verrons plus loin, des indices non zoologiques tendent à indiquer une préférence pour les bovidés, les suidés et les gallinacés - qui ne seront évoqués que de façon marginale. Quant aux ovicaprinés, ils sont plus présents grâce aux vestiges des productions qui leur sont liées - tissu et fromage - que pour la consommation de leur viande. À partir des données recueillies par des études menées essentiellement en Gaule, mais aussi, quoique de façon plus synthétique, en Italie, il est possible de restituer la chaîne opératoire générale de la boucherie, sans toutefois prendre en compte ni les lieux, ni les acteurs de ces différentes phases de travail. ${ }^{1}$ En effet, si les analyses archéozoologiques permettent, dans le meilleur des cas, de déchiffrer partiellement le palimpseste mutilé que constituent les dépôts d'os découverts en fouilles, il est souvent délicat de déterminer si le débitage d'un os en tronçon a été suivi ou précédé du désossage. Il ne sera question ni de la première phase de boucherie - l'abattage -, ni du contexte potentiellement religieux de celle-ci. Pour les différents mammifères, les sous-produits de la boucherie sont les premiers à être enlevés : le sang, éventuellement recueilli ; la peau, retirée par ablation des autopodes ; les viscères, propres ou non à la consommation ; la tête, pouvant elle aussi devenir source de produits charcutiers ou de matière première pour le travail de la corne. ${ }^{2}$ Sans entrer dans les détails de découpe propres à chaque sous-espèce, je me contenterai de souligner - tout en simplifiant de façon outrancière - que les membres sont les premiers à être déposés ou désarticulés, avant que le

1 En plus de l'article de Sébastien LEPETZ dans le présent volume, pour la Gaule, on se réfèrera à Martine LEGUILLOUX, "Note sur la découpe de boucherie en Provence romaine", Revue Archéologique de Narbonnaise, vol. 24 (1991), pp. 279-288; Sébastien LEPETZ, "Lanimal dans la société gallo-romaine de la France du nord", Revue archéologique de Picardie numéro spécial 12 (Amiens, 1996), pp. 139-145 ; Yves LIGNEREUX, Joris PETERS, "Techniques de boucherie et rejets osseux en Gaule romaine", Anthropozoologica, vol. 24 (1996), pp. 45-98, part. pp. 58-69. À propos des pratiques de boucherie en Italie, on consultera Michael MACKINNON, "Production and consumption of animals in Roman Italy: integrating zooarchaeological and textual evidence", Journal of Roman Archaeology, Supplementary series 55 (Ann Arbor, 2004), pp. 163-171.

2 Sur la charcuterie, voir Martine LEGUILLOUX, "À propos de la charcuterie en Gaule romaine. Un exemple à Aix-en-Provence tronc ne soit débité en deux ou trois quartiers. Les traces relevées sur les os, quelle que soit l'espèce, indiquent l'utilisation de deux types d'instruments, successivement ou concurremment: le couperet qui permet le débitage franc des os et le couteau, employé soit pour certaines opérations liées à la première coupe, soit pour le désossage. [171] Avec des variations en fonction des régions et surtout des contextes, selon une distinction ville / campagne, c'est le couperet qui aurait été le plus employé. ${ }^{3}$ Pour l'Italie, M. MacKinnon souligne également cet emploi important dans les villes sous l'Empire. ${ }^{4}$

S'il convient de retenir la mise en évidence de deux grands types d'instruments de découpe, deux points doivent être pris en compte : d'une part, de façon unanime, les auteurs d'études archéozoologiques soulignent la difficulté de dresser une synthèse quant à l'emploi du couteau ${ }^{5}$; d'autre part, au même titre qu'il n'y a pas de méthode de découpe universelle - pas même pour une sous-espèce donnée - , il ne faudrait pas chercher un instrument unique utilisé tout autant pour démembrer un bœuf que pour fractionner la grille costale d'un agneau.

La représentation antique du travail de boucherie

Les représentations des bouchers au travail généralement sur des reliefs funéraires - permettent de préciser cette première approche des instruments utilisés dans le cadre de la découpe des animaux. Quelque treize représentations de couteaux, de bouchers au travail ou de personnel servile découpant de la viande permettent une rapide étude formelle sur ces instruments. Seul relief du $\mathrm{I}^{\mathrm{er}} \mathrm{s}$. av. J.-C. de ce petit corpus, la stèle funéraire de Cesena n'est pas à proprement parler une représentation de bouchers (fig. 1), faute de mention explicite de ce métier dans le texte de l'inscription. ${ }^{6}$ Toutefois,

(ZAC Sextius-Mirabeau)", Gallia, vol. 54 (1997), pp. 239-259, part. pp. 246-249.

3 Depuis le premier constat effectué par Isabelle RODETBELARBI, Jean-Hervé YVINEC ("Boucheries et dépotoirs de boucherie gallo-romains", Anthropozoologica, vol. 13 (1990), pp. 19-26, part. p. 24), qui soulignaient que 'l'usage du couperet est franche et massive, en particulier lors de la découpe du tronc', ces remarques sont régulièrement reprises lors de l'étude d'autres sites.

4 M. MACKINNON, "Production and consumption...", pp. 177-184.

5 Les traces liées à l'usage du couteau sont difficile à mettre en évidence et elles ne sont que rarement mentionnées dans les études les plus anciennes.

6 Corpus Inscriptionum Latinarum [désormais CIL] XI, 348 ; ILS 2269; Gerhard ZIMMER, Römische Berufsdarstellungen, DAI - Archäologische Forschungen 12 (Berlin, 1982), cat. n 8 pp. 99-100 ; Laura CHIOFFI, Caro: il mercato della carne nell'Occi- 


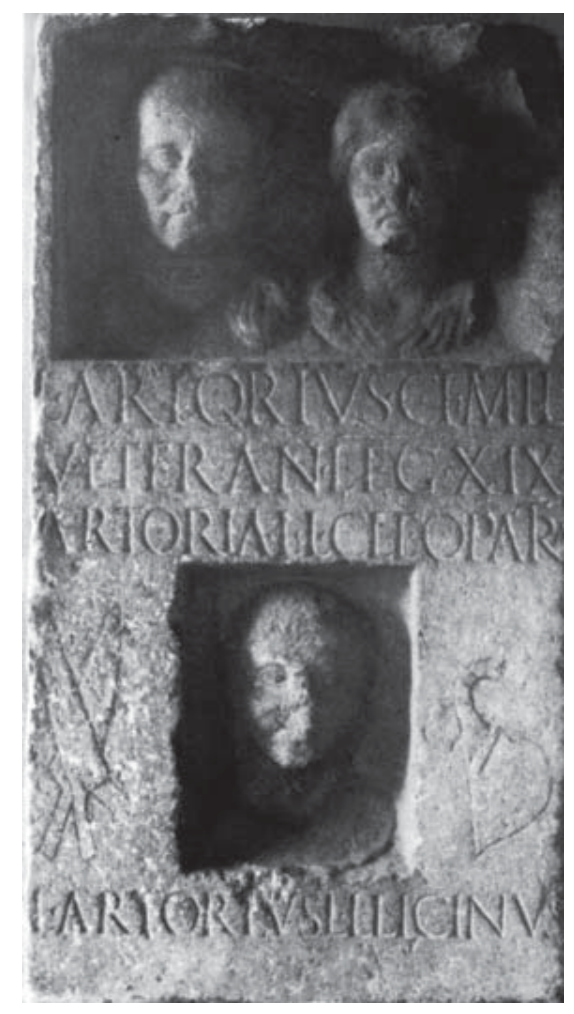

Fig. 1. Stèle funéraire de Cesena (Torcello, Museo Provinciale, inv. 7 ; Gerhard ZIMMER, Römische Berufsdarstellungen, DAI - Archäologische Forschungen 12, (Berlin, 1982), fig. 8 p. 100).

les couteaux représentés de part et d'autre du portrait de l'affranchi Lucius Artorius Licinus permettent d'avancer que les activités de ce dernier étaient liées à la découpe de la viande. À gauche de son portrait, deux couteaux à lame longue et droite, au dos incurvé sont croisés, manche vers le bas. De l'autre côté, est représenté un couperet à lame en demi-lune et dont le manche est coudé. Avant d'aller plus loin dans l'analyse iconographique de ces instruments de découpe [172] de la viande, il convient de signaler qu'un tel assemblage de couteaux est unique dans les représentations qui nous sont parvenues.

Une série de quatre reliefs propose également des représentations limitées aux seuls instruments, sans tentative de faire figurer l'espace de travail. Le premier, daté du I ${ }^{\mathrm{er}} \mathrm{s}$. de notre ère, provient d'Aquilée (fig. 2). Il s'agit de l'autel funéraire d'un affranchi qui ne

dente romano. Riflessi epigrafici ed iconografici, Atlante tematico du topografia antica - supplemento 4 (Roma, 1999), cat. $n^{\circ} 97$ p. 76.

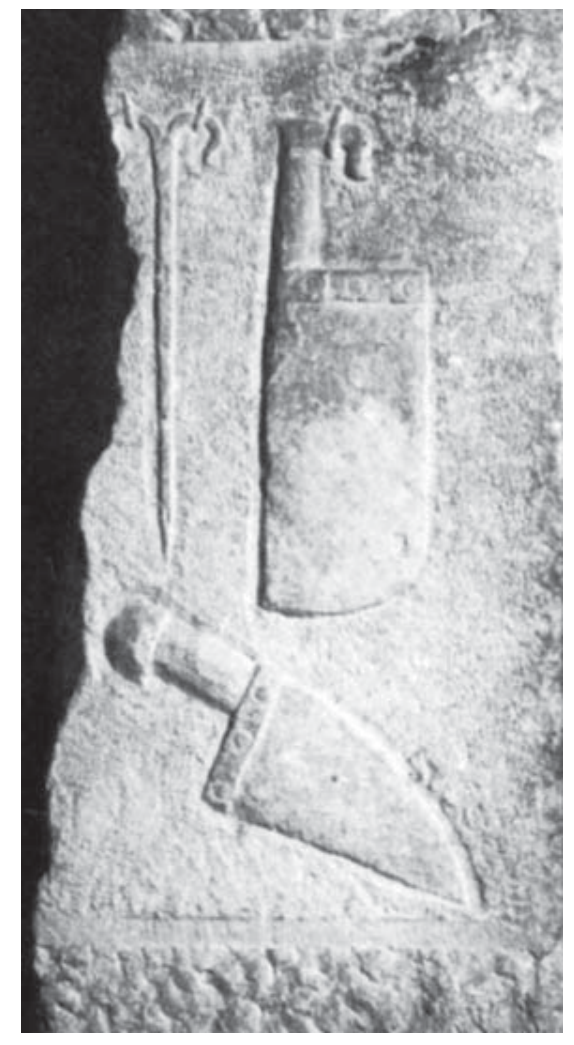

Fig. 2. Relief d'Aquilée (Museo Nazionale di Aquileia, inv. 448/1.2 ; G. ZIMMER, Römische Berufsdarstellungen..., fig. 13 p. 104).

mentionne pas explicitement son activité. Toutefois, deux outils de découpe en plus d'un double crochet à viande sont gravés sur le côté. ${ }^{7}$ La représentation est remarquablement détaillée, au point qu'il est possible de définir les aspects techniques de la conception de ces deux objets: pour l'un comme pour l'autre, le talon de la lame est enserré par une plaque rivetée qui constitue la continuité du manche. Ce sont deux types de couteaux qui sont représentés : le premier est un couperet à lame rectangulaire dont [173] seule l'extrémité est arrondie ; la feuille constituant le manche déborde légèrement sur le dos de la lame ; le second type est triangulaire et le tranchant est quasiment parallèle au manche. Bien que daté de façon extrêmement variable, entre le $\mathrm{I}^{\mathrm{er}}$ et le III ${ }^{e} \mathrm{~s}$. de notre ère, l'autel funéraire de Concordia Sagittaria présente de grandes ressemblances avec

7 CIL V, 1379 ; G. ZIMMER, Berufsdarstellungen..., cat. $\mathrm{n}^{\circ} 13$ p. 103 ; Y. LIGNEREUX, J. PETERS, “Techniques...”, p. 51 ; L. CHIOFFI, Caro..., cat. ${ }^{\circ} 104$ p. 80. 


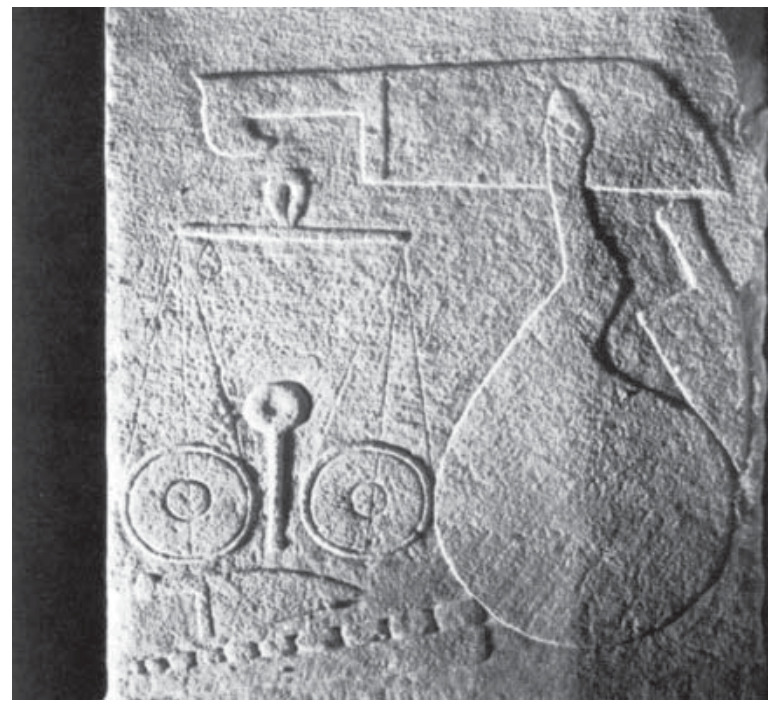

Fig. 3. Autel funéraire de Concordia Sagittaria (Museo Nazionale Concordiese ; G ; ZIMMER, Römische Berufsdarstellungen ..., fig. 14 p. 105).

celui d'Aquilée (fig. 3). ${ }^{8}$ Sur l'un des côtés, un long couperet à lame droite et deux couteaux à lame triangulaire sont disposés entre un jambon, une balance, des poids et un possible maillet. Le couperet et le plus petit des deux couteaux présentent un système d'emmanchement par enserrement de la lame, proche de celui de l'autel funéraire d'Aquilée, mais moins détaillé. Sur un relief provenant d'Amiternum, un tympan anépigraphe, sont figurés quatre instruments que l'on peut lier à la boucherie (fig. 4). ${ }^{9}$ On distingue ainsi une hache, un couperet, un couteau et un billot sur trois pieds. Deux d'entre eux peuvent être rattachés à la coupe primaire ou du moins à la découpe des os les plus gros, en percussion. En revanche, si l'on considère que ce relief est faiblement stéréotypé, ce à quoi la multiplicité des outils nous incite, il est possible d'interpréter l'utilisation du couteau non pour la découpe par percussion, mais plutôt pour le [174] désossage ou le débi-

8 G. ZIMMER, Berufsdarstellungen..., cat. $\mathrm{n}^{\circ} 14$ p. 104 ; Y. LIGNEREUX, J. PETERS, “Techniques...”, p. 51 ; L. CHIOFFI, Caro..., n. 6, cat. n 107 p. 83.

9 G. ZIMMER, Berufsdarstellungen..., cat. ${ }^{\circ} 12$ p. 103; Y. LIGNEREUX, J. PETERS, “Techniques...”, p. 51 ; L. CHIOFFI, Caro..., cat. n 89 p. 71.

10 L. CHIOFFI, Caro..., cat. n ${ }^{\circ} 92$ pp. $72-73$.

11 Émile ESPÉRANDIEU, Recueil général des bas-reliefs, statues et bustes de la Gaule romaine, Collection de documents

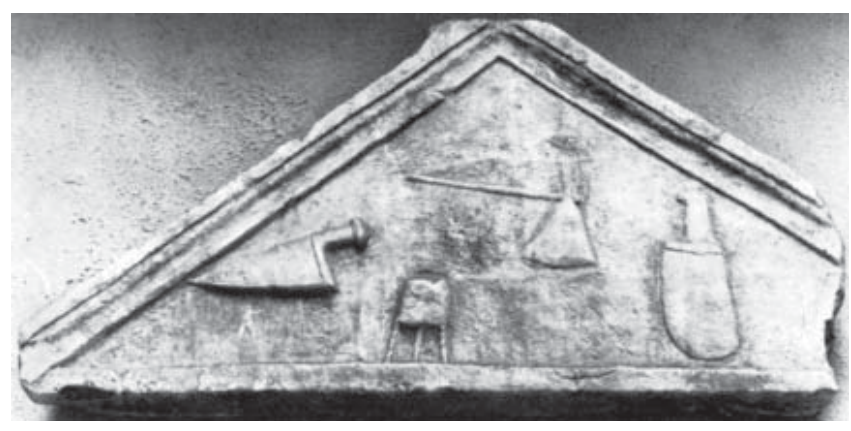

Fig. 4. Tympan anépigraphe d'Amiternum (L'Aquila, Museo Nazionale, sezione archeologica, inv. 100 ; G. ZIMMER, Römische Berufsdarstellungen..., fig. 12 p. 103).

tage de filets. Enfin, dans la partie inférieure d'une épitaphe très mutilée insérée dans un mur à Rieti, un cadre flanque un probable billot. ${ }^{10}$ Dans celui-ci, on reconnaît une hachette, un couteau à lame étrangement courbe et un récipient.

L'emploi de la hachette pour la coupe est attesté sur trois autres reliefs sur lesquels des scènes de travail ou du résultat de ce travail sont figurées. Le relief dit "du marchand de vin", provenant de TilChâtel, conservé à Dijon, est plus souvent utilisé pour sa représentation d'un vendeur de boissons (fig. 5). ${ }^{11}$ Toutefois, jouxtant le comptoir de ce dernier, se trouve un boucher dont l'étal présente des chapelets de saucisses regroupées par quatre ainsi que des hures pendues. À l'arrière, sur un billot, on aperçoit une hachette. Sur le relief de Trèves, c'est également un étal de boucherie qui est représenté (fig. 6) : côtes, jambons et tétines de truie sont disposés sur des présentoirs ou pendus. ${ }^{12} \mathrm{Au}$ premier plan, on voit deux hachettes, dont l'une prise dans un train de côtes disposé sur un billot [175], accompagnées d'un probable poinçon. Aucun de ces deux reliefs ne peut être daté avec certitude. En revanche, l'autel funéraire de C. Cornelius Successus, provenant d'Aquilée, est daté du Ier ou du IIe s. de notre ère (fig. 7) ${ }^{13} \mathrm{Il}$ représente ce dernier en train de

inédits sur l'histoire de France, tome IV (Paris, 1911), cat. n³608 ; L. CHIOFFI, Caro..., cat. n ${ }^{\circ} 140$ p. 102.

12 Heinz CÜPPERS, "Ein Metzger im römischen Trier", Trierer Zeitschrift, vol. 45 (1982), pp. 289-292 ; Y. LIGNEREUX, J. PETERS, “Techniques...”, p.54-56 ; L. CHIOFFI, Caro..., cat. n¹38 pp. $100-101$.

13 G. ZIMMER, Berufsdarstellungen..., cat. $\mathrm{n}^{\circ} 6$ p. 97; Y. LIGNEREUX, J. PETERS, “Techniques...”, p. 49 ; L. CHIOFFI, Caro..., cat. n॰105 pp. 81-82. 


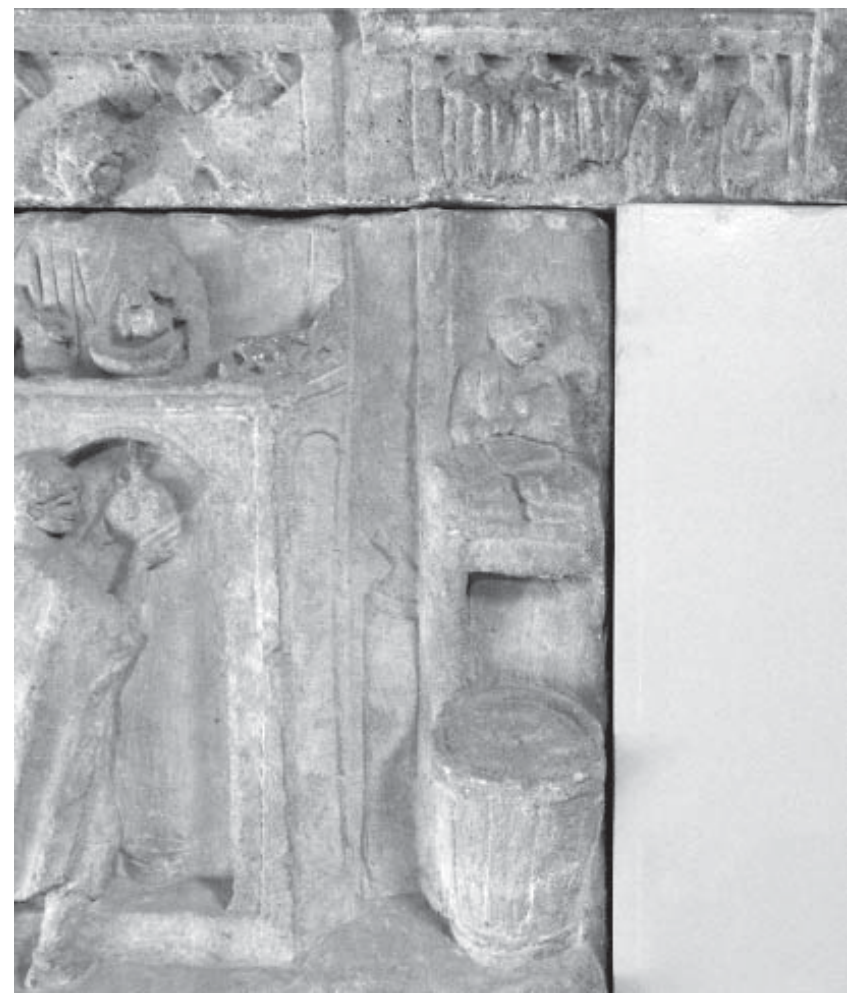

Fig. 5. Relief ‘du marchand de vin' de Til-Châtel (Musée archéologique de Dijon, inv. 138 ; photo N. Monteix).

fendre un porc pendu à un carnarium, une hachette est utilisée pour cette opération.

La représentation des scènes de découpe d'Italie tend à s'uniformiser durant le $\mathrm{II}^{\mathrm{e}}$ siècle ap. J.-C. si l'on se fie aux quatre reliefs qui ont été conservés ou dessinés (fig. 8). ${ }^{14}$ En dépit de légers détails pouvant être sujets à variation, leur organisation générale est étroitement similaire de l'un à l'autre. Le boucher, en tunique ou en toge est debout derrière un billot cylindrique ou juché sur trois pieds. Il tient à la main un couperet à large lame droite, levé, prêt à débiter une pièce de viande, qu'il s'agisse d'un train de côtes, d'un jambon ou d'une hure. Derrière lui se trouvent des crochets sur lesquels pendent systématiquement - sauf sur le dessin conservé au British

14 Relief du Trastevere, conservé à Dresde, voir G. ZIMMER, Berufsdarstellungen..., cat. $\mathrm{n}^{\circ} 2$ p. 94-95; Y. LIGNEREUX, J. PETERS, “Techniques...”, p. 51 ; L. CHIOFFI, Caro..., cat. nº 24 p. 34 .

Relief de Tiberius Iulius Vitalis, conservé à Rome, voir G. ZIMMER, Berufsdarstellungen..., cat. n 5 p. 96 ; Y. LIGNE-

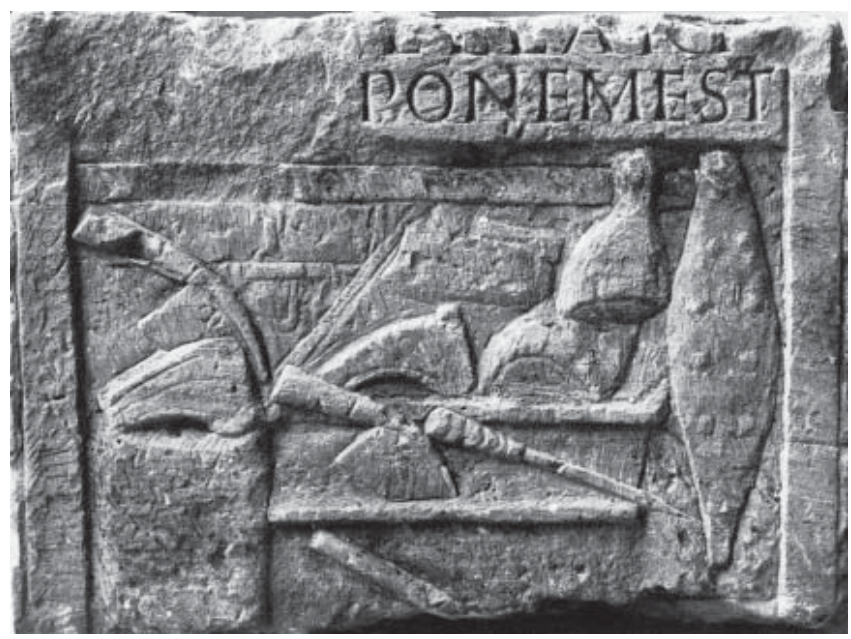

Fig. 6. Relief de Trèves (Heinz CÜPPERS, "Ein Metzger im römischen Trier”, Trierer Zeitschrfit, 45, 1982, fig. 1 p. 290).
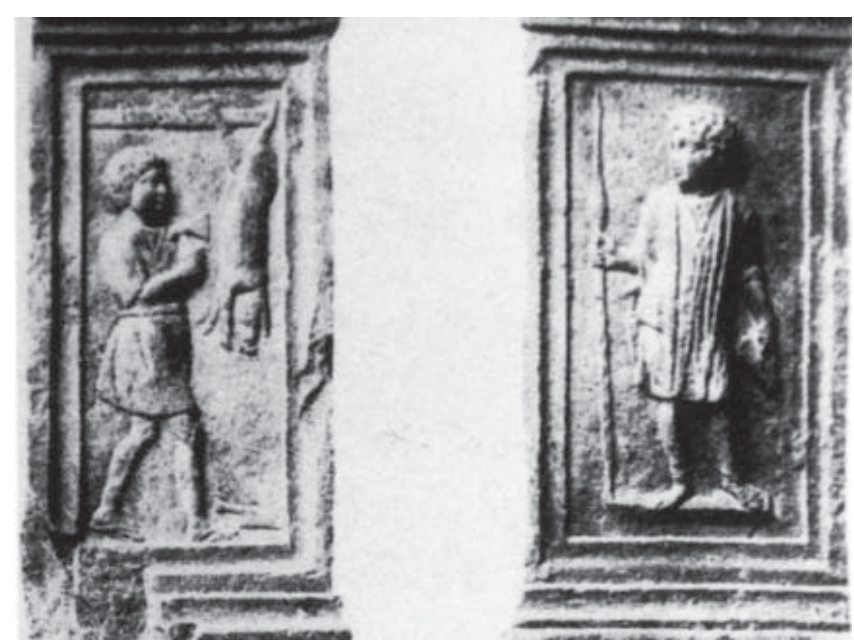

Fig. 7. Relief de Cornelius Successus provenant d'Aquilée (Laura CHIOFFI, Caro: il mercato della carne nell'Occidente romano. Riflessi epigrafici ed iconografici, Roma, 1999, fig. 48 p. 82).

REUX, J. PETERS, “Techniques...”, p. 54 ; L. CHIOFFI, Caro..., cat. $n^{\circ} 26$ p. 36-37.

Relief d'Ostie, voir G. ZIMMER, Berufsdarstellungen....., cat. n4 p. 95-96 ; Y. LIGNEREUX, J. PETERS, “Techniques...”, p. 54. Dessin de Dal Pozzo-Albani, conservé au British Museum, voir G. ZIMMER, Berufsdarstellungen..., cat. $\mathrm{n}^{\circ} 3$ p. 95 ; L. CHIOFFI, Caro..., cat. $\mathrm{n}^{\circ} 27$ p. 37. 

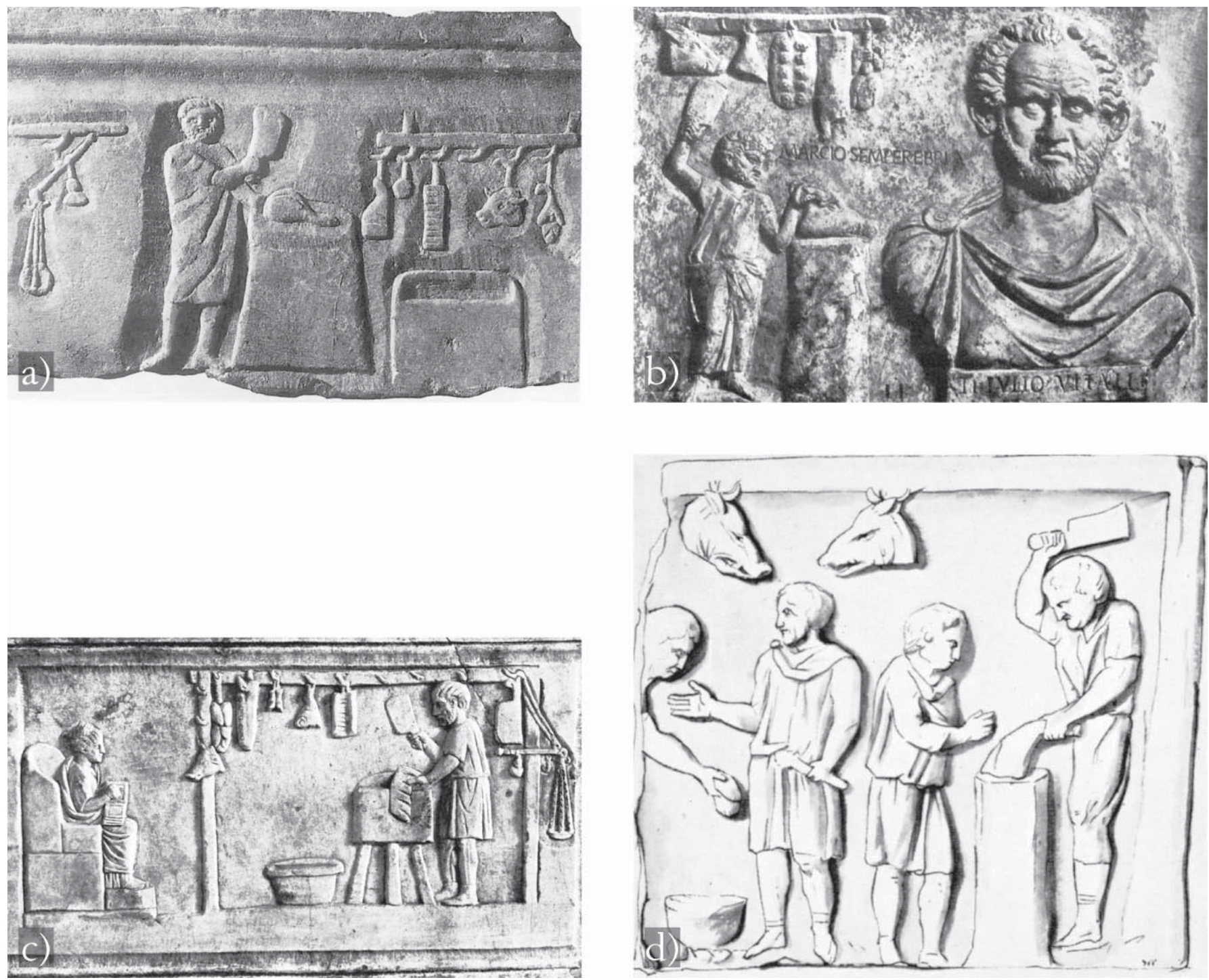

Fig. 8. Représentation de bouchers au travail. a) Relief funéraire d’Ostie (Museo Ostiense, inv. 133 ; Yves LIGNEREUX, Joris PETERS, “Techniques de boucherie et rejets osseux en Gaule romaine”, Anthropozoologica, 24, 1996, fig. 13 p. 54) ; b) Relief funéraire de Tiberius Iulius Vitalis (Rome, Villa Albani, inv. 11) ; c) Relief du Trastevere (Dresden, Skultpurensammlung, inv. 415) ; d) Dessin d'un relief de boucherie perdu (Londres, British Museum) (b-d, G. ZIMMER, Römische Berufsdarstellungen ..., fig. 2-4 p. 94-96).

Museum qui montre un relief mutilé - les principales pièces débitées dans des suidés : hure, tétines de truies, train de côtes, jambon, pieds et fressure. À proximité de ce carnarium - sauf sur le relief de Ti. Iulius Vitalis, lacunaire -, se trouve un bassin, récipient mobile ou probable aménagement construit, posé sur le sol. Il pourrait s'agir d'un réceptacle pour le sang des animaux abattus et découpés, ou pour les sous-produits de la coupe. Enfin, les deux reliefs complets présentent une balance, utilisée pour la vente au poids, sans distinguer la qualité des morceaux..$^{15}$ Soulignons que sur ces reliefs, le seul instrument de découpe qui est employé - ou qui est figuré accroché en réserve - reste le couperet à lame

15 Sur ce point, voir Martine LEGUILLOUX, "La boucherie et l'artisanat des sous-produits animaux en Gaule romaine", in J.-P. BRUN, Ph. JOCKEY (eds.), $\tau \varepsilon \chi v \alpha 1$. Techniques et sociétés en Méditerranée (Paris, 2001), p. 411-421, part. p. 412-413. 


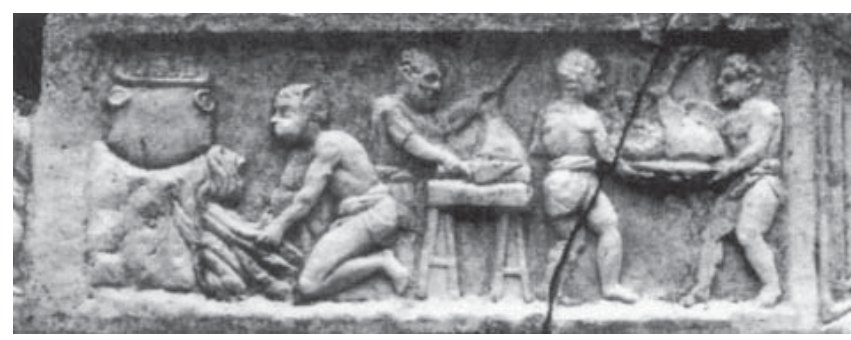

Fig. 9. Sarcophage de Tusculum (L. CHIOFFI, Caro..., fig. 30 p. 55).

rectangulaire et tranchant droit. ${ }^{16}$ Aucun couteau à lame triangulaire n'est représenté.

Enfin, la plus tardive des scènes de découpe connues se déroule dans une cuisine : sur un sarcophage de Tusculum daté du III ${ }^{\mathrm{e}}$ s., un commis [176] découpe un jambon tandis que deux autres emportent un plat sur lequel se trouve au moins une tête de cochon ou de sanglier (fig. 9). ${ }^{17}$ Le couteau utilisé pour tailler le jambon est à lame triangulaire.

Les reliefs qui viennent d'être évoqués permettent d'obtenir des précisions sur les types d'instruments employés par les bouchers romains. Toutefois, ces dernières restent limitées par l'emploi de certains codes de représentation. Ainsi, les scènes de coupe et de découpe représentent exclusivement des suidés, même sur le relief d'Ostie pour lequel la hure pourrait être interprétée comme une tête de veau. ${ }^{18}$ Malgré certains détails d'un 'réalisme' frappant, en particulier le mode d'assemblage entre le manche et la lame sur le relief d'Aquilée, il est très probable que des codes iconographiques aient également été employés pour les couteaux. Ainsi, deux

16 On indiquera toutefois l'exception de l'épitaphe de L. Pacuvius Restitutus et de Cassia Spicula, datée du IIe siècle, provenant des environs de Rome (G. ZIMMER, Berufsdarstellungen..., cat. $n^{\circ} 16$ p. 106 ; L. CHIOFFI, Caro..., cat. ${ }^{\circ} 21$ p. 32 ), où c'est un couteau à tranchant arrondi qui est représenté schématiquement, entouré à gauche par un jambon et à droite par une hure. En dépit du contexte chronologique, il ne saurait être question de rapprocher cette stèle des reliefs montrant des scènes de boucherie.

17 L. CHIOFFI, Caro..., cat. ${ }^{\circ} 55$ p. 55.

18 L'identification de la tête suspendue peut prêter à confusion : la première impression permettrait de la considérer comme un veau (Y. LIGNEREUX, J. PETERS, “Techniques...”, p. 54). Toutefois, la présence sur le relief, à gauche de la tabula ansata qui contient l'ensemble, de deux truies, permet de suivre G. ZIMMER (Berufsdarstellungen..., p. 96) et d'y voir une hure.

19 Le premier se trouve sur le relief du laraire de la maison de Caecilius Jucundus $(\mathrm{V} 1,23)$ : à droite d'un boeuf mené vers le sacrifice, un couteau est représenté. Le second est le tympan du laraire situé dans l'atrium v de la maison des Vettii (VI 15, 1.27) représentations provenant de Pompéi, issues de contextes éminemment religieux, créent une association entre le sacrifice et un couteau schématique à lame triangulaire ${ }^{19}$ En dépit de cette remarque de prudence, cette rapide étude iconographique permet de conclure à l'utilisation de trois instruments pour la coupe et la découpe : la hachette, le couperet à lame rectangulaire et le couteau à lame triangulaire [178].

\section{L'exemple de Pompéi : attestations du commerce de la viande}

À Pompéi, la situation est moins idéale que ce que la réputation du site veut bien lui prêter. Ainsi, les dépôts d'os rejetés et recouverts par l'éruption, donc correspondant aux dernières années de vie de la cité, se réduisent au nombre de trois. ${ }^{20}$ Le premier, découvert en octobre 1899 et décrit par A. Sogliano se situe dans une officine de lavage des toisons (VII 9, 43) insérée dans l'espace résiduel à l'est du sanctuaire consacré à la domus diuina, au sud du macellum. ${ }^{21}$ Non inventorié, il faut considérer ce dépôt comme perdu. À partir de 1994, une équipe japonaise a fouillé l'extérieur de la muraille, à la recherche de la Porta Capuana. Un dépôt d'os y a été découvert, immédiatement sous les strates déposées par l'éruption du Vésuve. L'étude est toujours en cours, seule la présence des différentes espèces a été publiée en 1996 : des restes de canidés, équidés, cochons, cervidés, boeufs et chèvres ont été mis au jour. $^{22}$ Enfin, lors de la campagne 2005 des fouilles entreprises par le Centre Jean-Bérard dans la tannerie de Pompéi (V 5, 2), un important dépôt de

qui présente, en stuc, une patère entourée d'un bucrane à gauche et d'un couteau à droite.

$20 \mathrm{La}$ synthèse la plus complète sur la faune pompéienne, qu'il s'agisse d'animaux de boucherie ou non, a été proposée par Anthony KING, "Mammals. Evidence from wall paintings, sculpture, mosaics, faunal remains, and ancient literary sources", in W.F. JASHEMSKI, F.G. MEYER (eds.), The natural history of Pompeii (Cambridge, 2002), p. 401-450. Outre deux des trois dépôts à peine évoqués, il en mentionne plusieurs autres, dont certains non publiés, datant des périodes antérieures au Ier s. de notre ère, qui ne peuvent être retenus dans le cadre de cette étude.

21 Notizie degli scavi di Antichità, 1899, p. 390 : "Nello strato antico di terra che ricopriva il chiassuolo A si rinvenne una grande quantità di ossa di quadrupedi, come mandibole di vaccina, corna di bufalo, e di cerviatto, mascelle di altri animali”. Sur ce dépôt d'ossements animaux voir, dans ce volume, la contribution de William VAN ANDRINGA.

22 Makoto WATANABE "The natural remains unearthed from UU.SS.4”, Opuscula Pompeiana, vol. 6 (1996), pp. 63-65. 


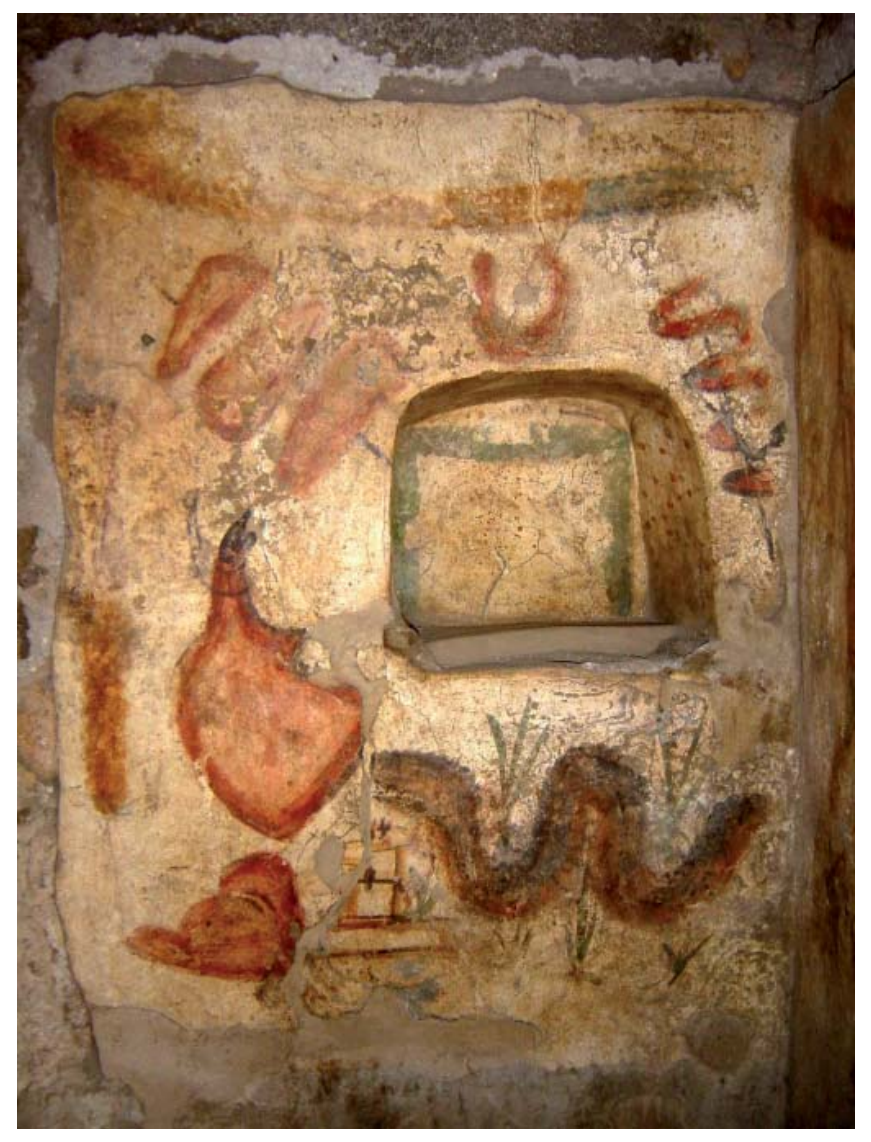

Fig. 10. Fresque représentant des morceaux de porc autour d'une niche du culte domestique dans la maison I 13, 2 (photo W. van Andringa)

pattes de moutons sectionnées a été découvert en association avec du matériel écarté lors du fonctionnement de ce local. Ces rejets liés aux premiers sous-produits de l'abattage des moutons ne livrent malheureusement pas d'information sur les pratiques de boucherie stricto sensu. ${ }^{23}$

Si ces différents problèmes de conservation ou d'étude empêchent de percevoir le travail des bouchers par le biais de leurs déchets, d'autres sources nous permettent d'éclairer leurs productions. Ainsi, plusieurs peintures montrent très nettement les différentes formes d'accommoder la viande de cochon, autant de témoignages qui ne peuvent que traduire une pratique courante. Les deux premiers exemples

23 Martine LEgUILlOUX, "L'artisanat du cuir à Pompéi", Mélanges de l'École française de Rome - Antiquité, vol. 118-1,

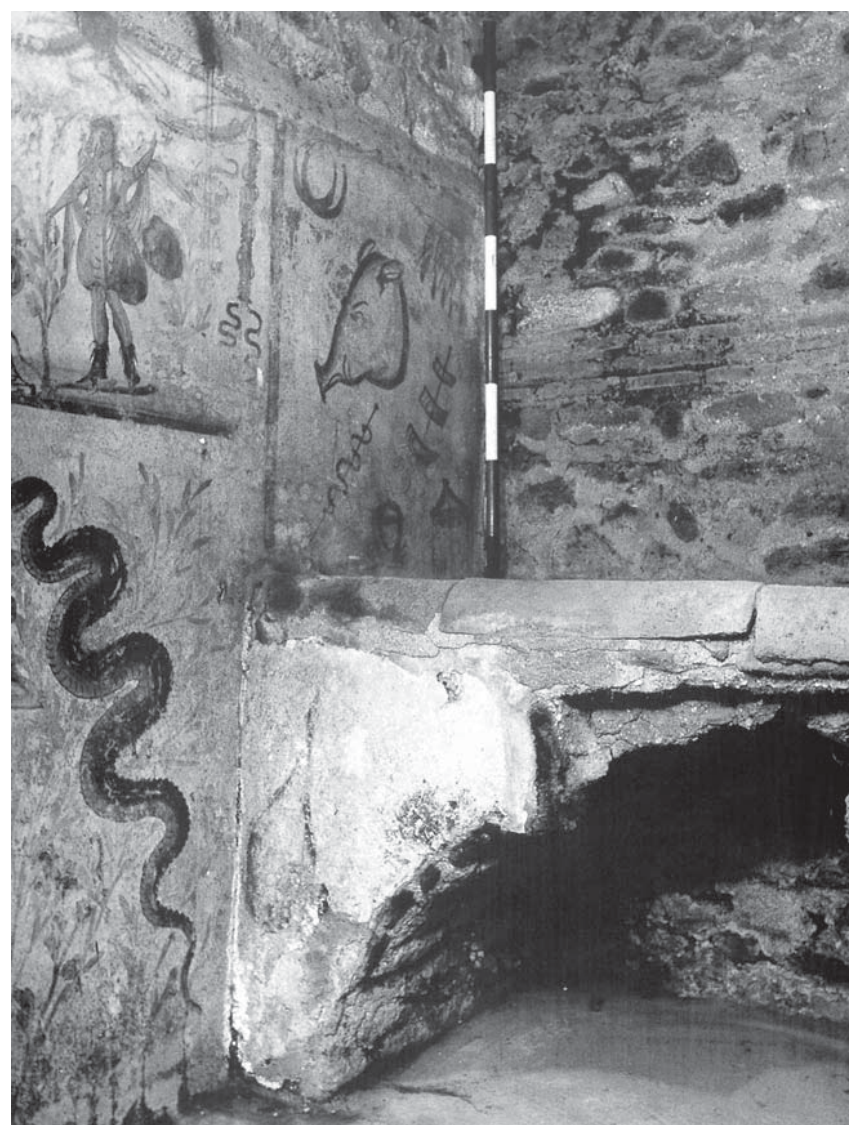

Fig. 11. Fresque représentant des morceaux de porc entre un autel du culte domestique et un fourneau de cuisine dans la maison IX 9, c (Pompei. Pitture e mosaici, vol. X, Roma, 2003, fig. 49 p. 29).

sont liés à des autels du culte domestique situés dans des cuisines. Dans la maison dite de Sutoria Primigenia (I 13, 2), un jambon, un chapelet de saucisses, une [179] saucisse embrochée et trois morceaux de viande embrochés sont peints autour de la niche cultuelle (fig. 10). Dans la maison dite de C. Sulpicius Rufus (IX 9, c), on retrouve les mêmes éléments, auxquels s'ajoutent une hure et un jambon : cette fresque liée au culte domestique est peinte sur la paroi contre laquelle s'appuie le fourneau de la cuisine (fig. 11). Le troisième exemple est d'autant plus intéressant qu'il ne provient pas d'un espace domestique mais du commerce alimentaire et restaurant situé en VI 10, 1.19 : au-dessus des clients attablés 


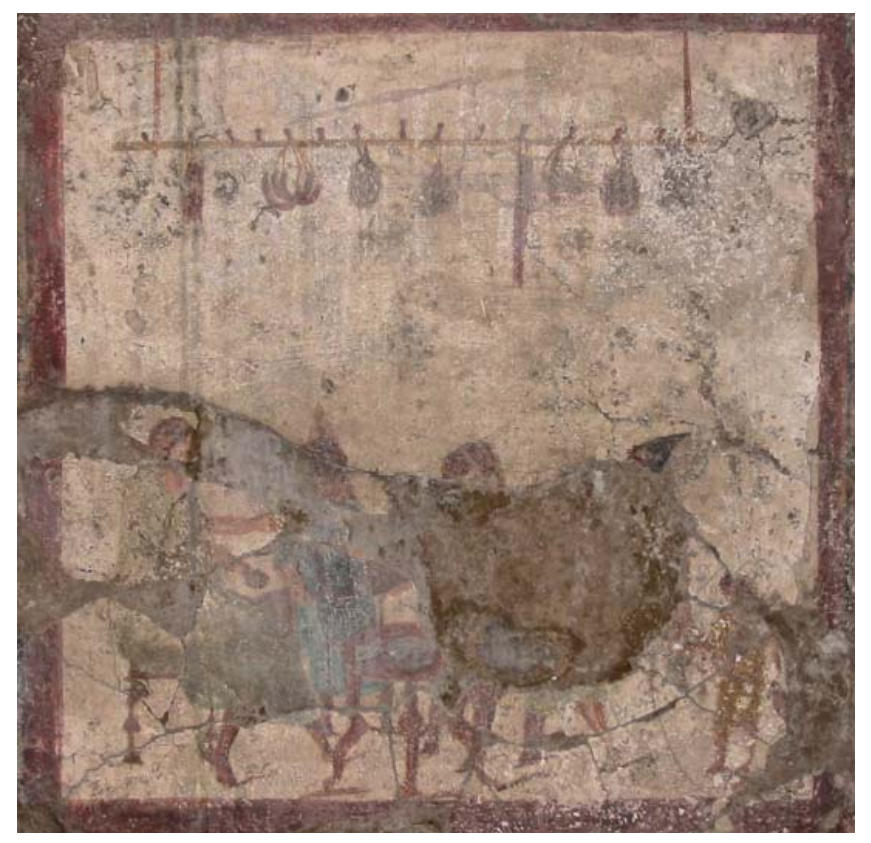

Fig. 12. Fresque représentant les clients d'un restaurant avec de la charcuterie séchant au-dessus d'eux (Pompéi, VI 10, 1.19 ; photo N. Monteix).

pendent des chapelets de saucisses et de probables saucissons (fig. 12). ${ }^{24}$

Une seconde forme d'attestation du commerce de la viande provient des graffitis laissés sur les murs de la ville. Une série de huit listes indiquant parfois des prix a été retrouvée dans divers points de la ville. ${ }^{25}$ [180] Trois d'entre elles mentionnent de la viande. La première provient du commerce alimentaire, restaurant et peut-être hôtel, IX 7, 24-25. ${ }^{26}$ Elle comprend, en trois colonnes inégales, une liste de

24 Traditionnellement appelés 'thermopolia', ce type de commerce alimentaire devrait, si l'on veut vraiment employer un terme latin, être appelé caupona. Voir Nicolas MONTEIX, "Cauponae, popinae et 'thermopolia', de la norme littéraire à la réalité pompéienne", Contributi di Archeologia Vesuviana. III, Studi della Soprintendenza Archeologica di Pompei 20 (Roma, 2007), pp. 115-126.

25 CIL IV, 4000 ; 4227 ; 4422 ; 4888 ; 5380 ; 6263 ; $8561 ; 8566$.

26 CIL IV, 5380. Le texte de cette inscription est reporté en annexe.

27 CIL IV, 8561. Graffite lu sur la colonne XVIII, située dans la branche méridionale du portique de la palestre. Le texte de cette inscription est reporté en annexe.

28 CIL IV, 8566, b. Graffite sur la même colonne que l'inscription CIL IV, 8561. Le texte de cette inscription est reporté en annexe. produits répartis selon les jours écoulés entre le septième jour avant les ides et les ides. Après chaque denrée, un prix est indiqué en as, parfois en deniers. Aucun élément ne permet de déterminer de façon définitive s'il s'agit d'une liste d'achats ou de produits vendus dans le commerce situé en IX 7, 24. Une seule fois au cours des huit jours mentionnés, de la viande est indiquée : 'bubella' (à la place de bubula, viande de boeuf) et botellum (saucisse ou boudin), pour une valeur d'un as. Deux autres listes provenant de la Palestre indiquent également le prix de la viande. La première mentionne le prix d'une livre de lard (3 as) et de viande de porc (4 as). ${ }^{27} \mathrm{La}$ seconde mentionne également le porc, pour une valeur d'un as, et probablement du boeuf, pour une valeur identique. ${ }^{28}[181]$ Cette seconde inscription de la palestre a été interprétée comme une liste de courses. Toutefois, une nouvelle interprétation d'un signe illisible permet de comprendre cette liste comme un compte progressivement soldé, en deux versements, ce qui indiquerait un emplacement de commerce alimentaire ambulant, vendant non seulement de la viande, mais également les principaux produits de l'alimentation pompéienne : pain, vin, huile et fromage. ${ }^{29}$

Une dernière attestation du commerce de la viande doit être signalée. Des gallinari, volaillers, sont ainsi cités sur les affiches électorales. ${ }^{30} \mathrm{Si}$ la mention de corps de métier sur ce type d'inscriptions n'est plus interprétée comme le signe de l'existence de collèges ou d'une démocratie vivante, elle souligne toutefois la présence de ces métiers dans la ville. L'hypothèse qui prévaut désormais, fortement suggérée par H. Mouritsen puis développée par C. Chiavia, est que les rogatores, personnes ou groupes de personnes demandant l'élection de certains candidats, auraient été payés par ceux-ci. ${ }^{31}$ Dans le cas des gallinari, il paraisse raisonnable d'avancer qu'ils

29 L'identification des produits de la liste comme ayant été achetés sur le marché de Pompéi a été proposée par Matteo DELLA CORTE (Notizie degli Scavi di Antichità [désormais NSc] 1939, p. 250). Avec cette hypothèse, on ne s'explique pas la mention de deux versements: utiliser une colonne pour se souvenir de sommes reçues paraît superflu, à moins que l'on ne revienne régulièrement exercer une activité en ce point.

30 CIL IV, 241 et 373.

31 Henrik MOURITSEN, Elections, magistrates and municipal élite. Studies in Pompeian Epigraphy, Analecta romana instituti danici - supplementum 15 (Roma, 1988), pp. 60-68; Henrik MOURITSEN, "Electoral campaigning in Pompeii: a reconsideration”, Athenaeum, vol. 22 (1999), pp. 515-523 ; Catherine CHIAVIA, Programmata. Manifesti elettorali nella colonia romana di Pompei (Torino, 2002), pp. 237-245. 
devaient être suffisamment nombreux pour que leur soutien semble lié à une partie non négligeable de la population, quand bien même il ne s'agirait que de basse propagande électorale, effectuée avec la collaboration de seulement quelques individus. Une partie du commerce de la volaille pourrait avoir été effectuée dans le macellum, comme l'illustre une fresque dans le monument, et dans les commerces alimentaires comme en témoigne le restaurant dit 'de Pherusa' (III 6, 1-3), en devanture duquel un probable enclos à volailles en terre crue a été mis au jour. ${ }^{32}$ En revanche, aucun matériel de découpe ne provient de cette boutique.

\section{Les couteaux de bouchers à Pompéi : problèmes de conservation}

Si ces différents exemples indiquent quelques éléments liés au commerce et à la consommation de la viande à Pompéi, ils ne permettent pas de mettre en évidence ni le lieu de la boucherie, ni son organisation. Pour tenter [182] d'aboutir à des conclusions sur ce point, j'ai commencé un dépouillement exhaustif des archives des fouilles menées à Pompéi pour en extraire les localisations des couteaux, outils indispensables à la boucherie. Les inventaires dressés durant les fouilles et les éventuelles descriptions liées à ces découvertes sont partiellement publiés, tandis que les collections sont éparpillées entre le Musée archéologique de Naples, le site de Pompéi et diverses collections privées ou nationales à travers le monde (fig. 13). ${ }^{33}$

Une fois ces archives clairement identifiées et dépouillées, deux problèmes apparaissent. Le premier est celui de l'appellation donnée aux couteaux au moment de leur inventaire. Si de nombreux 'coltelli' peuvent être identifiés, certains s'avèrent avoir eu une toute autre fonction lors de leur observation directe. C'est ainsi le cas d'un objet découvert dans la boutique V 4, 3 en 1899: décrit comme un "pic-

32 NSc 1936, p. 311.

33 Les principales éditions des archives des fouilles de Pompéi sont les suivantes: Giuseppe FIORELLI (ed.), Pompeianarum antiquitatum historia quam ex cod. mss. et a schedis diurnisque... (Napoli, 1860-1864) (PAH, pour la période 1748-1860); Giornale degli Scavi di Pompei, 1850-1851, 1861-1865, 1868-1879 (GSPomp); Atti della (reale) Accademia (nazionale) dei Lincei. Notizie degli Scavi di Antichità, 1876- (NSc, à partir de 1876, régulièrement jusqu'en 1917, puis seulement de façon épisodique à partir de 1923). Les retranscriptions manuscrites des journaux de fouille sont intégralement conservées à partir de 1860 à colo coltello privo della punta", son examen a en fait démontré qu'il s'agissait de forces ayant perdu la seconde lame et la majeure partie du ressort. ${ }^{34}$ Inversement, bien que cela soit plus rare, certains objets sont affublés de noms qui n'ont rien à voir avec leur fonction réelle. Ainsi, trois grands types d'objets considérés comme des scalpelli ont été mis en évidence : il s'agit soit de ciseau - à bois ou à pierre ce qui correspond au nom donné ; soit d'un cutter / scalpel, petite lame de découpe très maniable; soit d'un couperet. Ces appellations variables obligent à effectuer un examen direct de tous les couteaux et autres objets s'approchant, de façon à pouvoir définir précisément leur forme et leur fonction. C'est ici qu'un autre problème, lié à l'histoire du site depuis sa redécouverte en 1748 se pose : tous les objets ne sont pas conservés dans les dépôts à Pompéi. Si la majeure partie des objets découverts entre 1748 et 1860 a été d'abord exposée dans le Reale Museo Borbonico de Portici puis versée après 1860 dans les collections du Museo Archeologico Nazionale de Naples (MANN), certains ont pu être offerts en cadeau aux personnalités visitant les fouilles. Entre 1861 et 1905, la plupart des objets continue d'être envoyée au musée. Toutefois, certains sont exposés dans l'Antiquarium ou sur le lieu de leur découverte. Après 1905, sauf exceptions de grande valeur, tous les objets restent à Pompéi [183], pour être conservés soit dans les dépôts du site, soit dans l'Antiquarium. En plus de cet éparpillement, une partie des objets conservés à Pompéi a été détruite lors du bombardement allié de 1943 : une bombe est notamment tombée sur le 'Museo Pompeiano', réduisant en poussière quelque 31 couteaux pour ne pas mentionner les autres objets. ${ }^{35}$ Enfin, à ces dommages collatéraux, s'ajoutent les pertes de données créées entre la fin des années 1970 et le début des années 1980, période à laquelle tous les objets conservés sur leur lieu de découverte ont été déplacés vers les dépôts du site, sans que leur provenance originelle soit notée.

Pompéi dans les locaux de la Surintendance. Des lacunes assez nombreuses existent à partir des années 1950.

34 Inv. n 54118 (ex 1804), découvert le 30 avril 1899. Le changement de forme de la section de la 'soie', qui passe d'une section quadrangulaire verticale à une section quadrangulaire horizontale, indique qu'il s'agit en fait de l'amorce d'un ressort de force, brisé.

35 Sur les conséquences à Pompéi du bombardement de 1943, voir Laurentino GARCÍA Y GARCÍA, Danni di guerra a Pompei. Una dolorosa vicenda quasi dimenticata, Studi della Soprintendenza Archeologica di Pompei 15 (Roma, 2006), pp. 19-29, 201-205. 


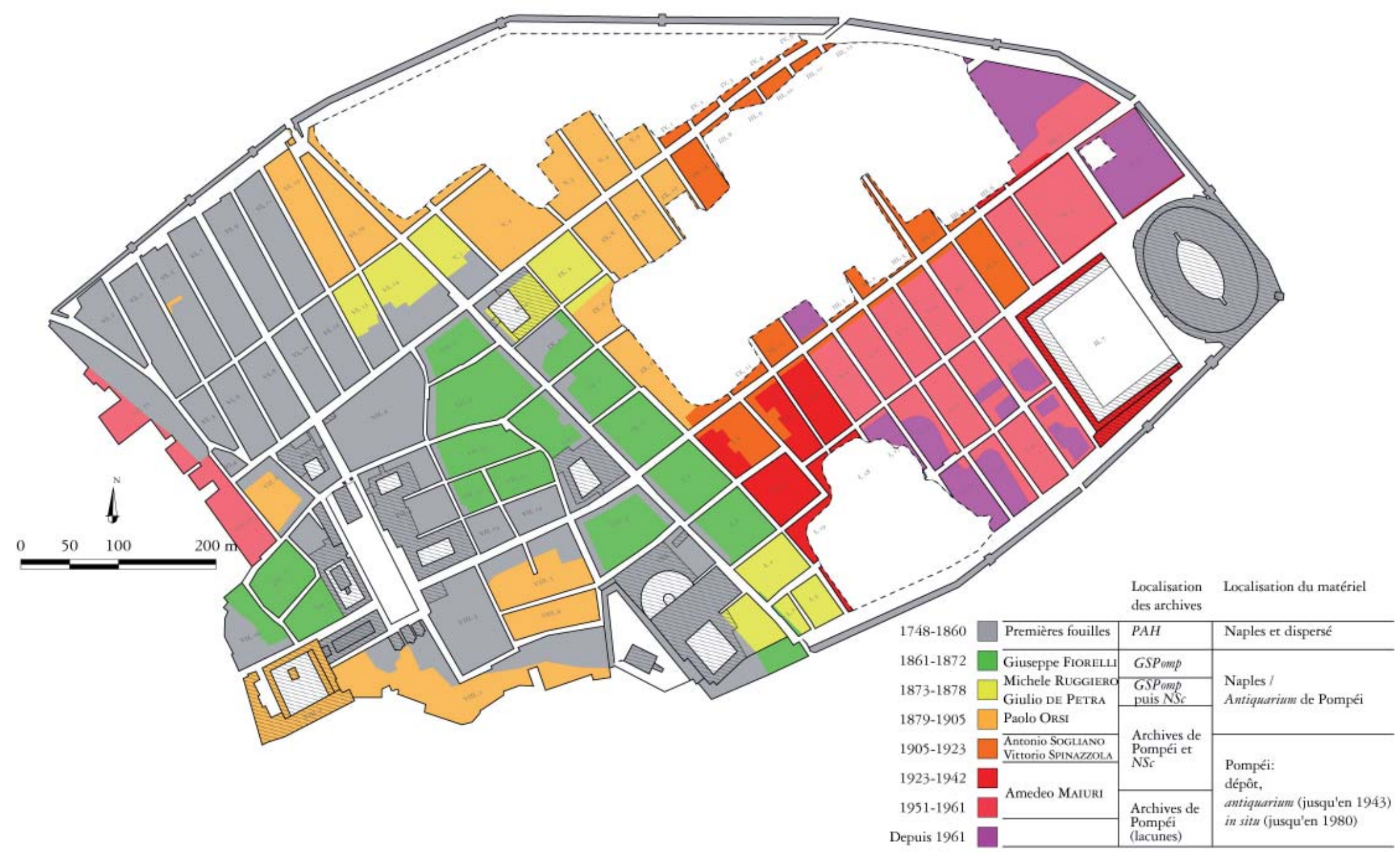

Fig. 13. Chronologie sommaire des fouilles de Pompéi et localisation des objets découverts durant celles-ci (cartographie N. Monteix). 
En l'état actuel, le dépouillement et l'observation directe des couteaux restent partiels. La PAH n'a été consultée que de façon liminaire, pour suivre le déroulement de la fouille du macellum, dans lequel aucun instrument de découpe n'a été mis au jour selon les archives publiées par Giuseppe Fiorelli. Pour la période allant de 1861 à 1878 , des décomptes généraux ont été publiés : 160 couteaux ou considérés comme tels ont été découverts entre 1861 et 1872, dont 4 indiqués comme 'coltellaci'. ${ }^{36}$ Entre 1873 et 1878, seuls 67 instruments tranchants ont été mis au jour, dont 3 'coltelloni '. ${ }^{37}$ Lanalyse des contextes de découverte est encore en cours. Le principal problème risque d'être lié à l'observation des couteaux, majoritairement si ce n'est exclusivement conservés au MANN. Entre 1879 et 1980, 153 couteaux ont été inventoriés. Sur les 113 encore conservés à Pompéi, 75 ont été étudiés - la majeure partie des objets restant étant constituée de forces. À chaque fois que cela a été possible, ont également été ajoutés les couteaux détruits durant le bombardement mais photographiés au préalable. En dépit des aspects éminemment partiels de cette étude, certains caractères émergent et permettent de dresser un premier cadre général.

En se fondant sur les analyses archéozoologiques provenant d'autres sites, qui indiquent l'emploi de couperet pour couper les os et de couteau pour désosser les carcasses ou découper la chair, et en s'appuyant sur les représentations iconographiques déjà mentionnés, nous pouvons écarter un certain nombre d'objets comme ne relevant pas de la boucherie. Ainsi, les ciseaux à bois et à pierre avérés, les forces, les rasoirs pliables et les petits canifs ont été exclus. Je ne détaillerai pas la typologie des couteaux qu'il est possible de dresser sur des critères morphologiques (longueur [185] et forme de la lame) et techniques (nombre de pièces employées, type d'emmanchement). En analysant les contextes de découverte, il est possible de mettre

36 Giuseppe FIORELLI, Gli scavi di Pompei dal 1861 al 1872. Relazione al ministro della Istruzione Pubblica (Napoli, 1873), pp. 169-171.

37 Luigi VIOLA, "Gli scavi di Pompei dal 1873 al 1878", Pompei e la regione sotterrata dal Vesuvio nell'anno LXXIX, Parte seconda (Napoli, 1879), pp. 7-85, part. 80-84.

38 Les six lames découvertes le 15 février 1913, derrière le montant oriental de la boutique I 9, 2, peut-être rangées dans une armoire en bois, ont été inventoriées sous le numéro 1706 (détruites lors du bombardement de 1943) et décrites comme suit dans le livre d'inventaire : "Curioso e nuovo coltello stretto verso l'impugnatura e largo al taglio come ventaglio, alto m. 0,30. L'im- en évidence une série de couteaux qui peuvent raisonnablement être considérés comme liés aux activités de boucherie. De cette façon, la plupart des couteaux que l'on peut considérer comme "d'office" - qui n'ont pas de fonction clairement définissable par leur forme et qui sont donc à usages multiples - ont été écartés quand ils n'ont pas été découverts en lien avec des couteaux interprétés comme de boucherie.

Une première série de couteaux a été découverte en dix exemplaires dans deux maisons différentes : six en I 9, 2 et quatre en I 13, 6 (fig. 14, a-b). ${ }^{38}$ Les six premiers ne sont plus étudiables, ils ont été détruits par les [186] bombes alliées. Toutefois, les photos publiées dans les Notizie degli Scavi di Antichita (désormais $N S c$ ) permettent de les identifier sans difficulté. Dans les deux cas, et bien que I 9, 2 soit une boutique, les couteaux ont été découverts groupés - attachés entre eux par l'oxydation dans le cas de I 13, 6 - rangés dans des armoires. Ils disposent d'une lame droite au tranchant en $\mathrm{V}$ arrondi, se terminant par une soie enserrée dans deux plaques d'os ou de bois qui servent de manche. La hauteur de la lame - $13 \mathrm{~cm}$ - correspond à la hauteur des rares couperets connus de forme quadrangulaire, conservés au British Museum ou découverts à Sierentz (fig. 15, a-b) ${ }^{39}$ De plus, la forme est extrêmement proche du couteau représenté sur la stèle funéraire provenant de Cesena. L'interprétation de ces dix couteaux comme des couperets, lourds, massifs, permettant de procéder à la coupe d'une carcasse à peine abattue, paraît être raisonnable. Si le contexte commercial de I 9, 2 est assez délicat à interpréter en raison de la disparition des journaux de fouille, il faut noter que la maison I 13, 6 n'est pas une boutique. De plus, l'examen attentif des couteaux montre qu'ils disposaient d'un fourreau en bois permettant un transport sans danger. Si l'on ajoute à cela l'exiguïté de cet espace, il nous faut considérer que les couteaux provenant de I 13, 6 étaient déposés

pugnatura risulta di due lamine accoppiate di bronzo finienti in codetta arcuata". Voir également NSc 1913, p. 61.

Les quatre lames de I 13, 6 ont été découvertes le 12 mai 1955, sans plus de précision quant à leur provenance dans l'ensemble concerné en raison de la disparition des journaux de fouille (Inv. $\mathrm{n}^{\circ} 11150$ ).

39 William H. MANNING, Catalogue of Romano-British irontools, fittings and weapons in the British Museum (London, 1985), cat. Q 97 p. 122 et pl. 57 ; Christian VALLET, "Les outils de la découpe des viandes à Sierentz", Cahiers de l'Association pour la promotion de la recherche archéologique en Alsace, vol. 11 (1995), pp. 73-88, part. pp. 82-83. 
a)

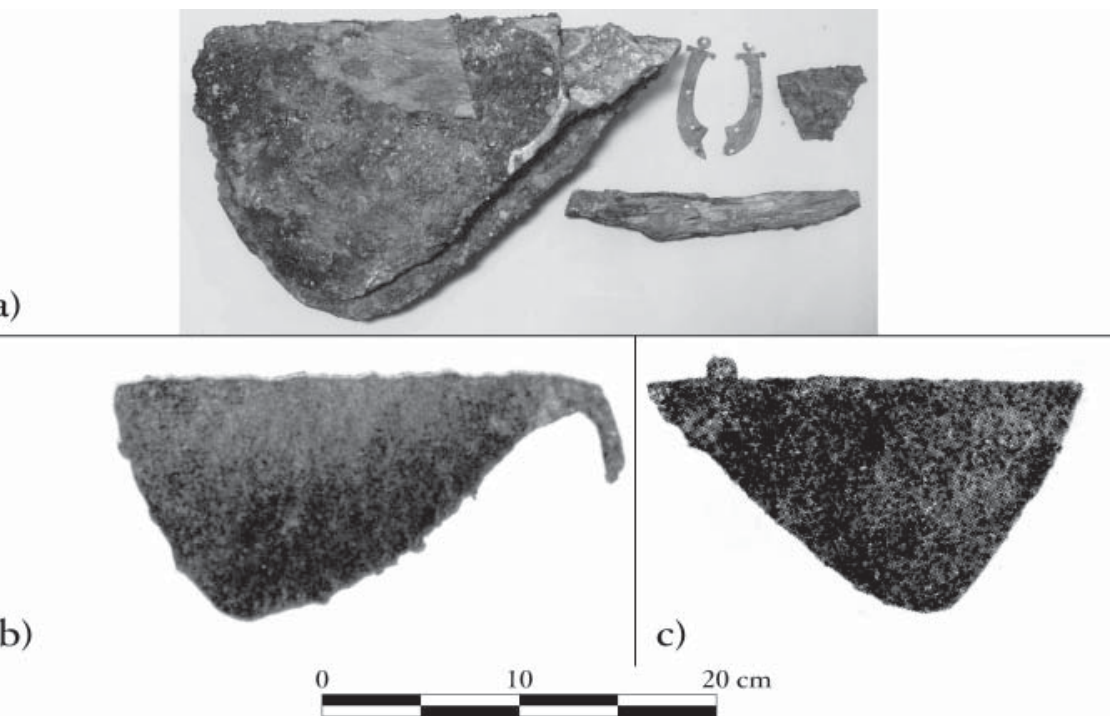

Fig. 14. Couperets de Pompéi (échelle 1/5). a) Quatre couperets associés par l'oxydation, découverts en I 13, 6 (Inv. 11150 ; photo N. Monteix) ; b) Couperet découvert avec cinq autres en I 9, 2 (Inv. 1706, détruit ; Notizie degli scavi di Antichità,

1913, fig. 5 p. 61) ; c) (Inv. 5169, détruit ; Notizie degli scavi di Antichità, 1934, fig. 28 p. 318).

a)

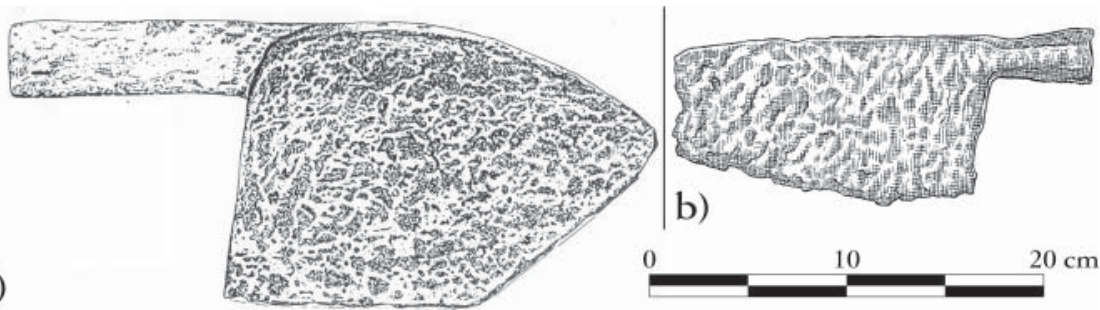

Fig. 15. Couperets (échelle 1/5). a) Couperet de Sierentz (Christian VALLET, "Les outils de la découpe des viandes à Sierentz", Cahiers de l'Association pour la promotion de la recherche archéologique en Alsace, 11, 1995, fig. 10 p. 83) ; b) Couperet de Kingsholm, Gloucestershire (British Museum ; William H. MANNING, Catalogue of Romano-British irontools, fittings and weapons in the British Museum, London, 1985, pl. 57).

au moment de l'éruption chez leur utilisateur, mais qu'il ne les employait vraisemblablement pas dans cet espace. Quant aux sept couteaux qui ont été mis au jour avec les six couperets en I 9, 2, s'ils doivent être liés à des activités de boucherie, ils peuvent être considérés comme des couteaux à découper des filets ou du moins à inciser, leur forme leur interdisant [187] d'être utilisés en percussion lancée pour découper des os (fig. 16, a). ${ }^{40}$ Là encore, leur aspect et leur association avec des couperets à lame arrondie permettent un intéressant parallèle avec la stèle funéraire de Cesena. Six autres exemplaires de ces couteaux proviennent de la maison I 7, 1 (fig. 16, b-c). Leur origine, un atrium, ne permet pas de confirmer ou d'infirmer leur rôle dans la boucherie, bien qu'ils soient probablement associés à deux couperets, ce qui permettrait alors d'interpréter ces huit lames de la même façon que les treize découvertes en
40 Sept lames découvertes le 15 février 1913, inventoriées sous le numéro 1705, détruites lors du bombardement de 1943. Description des livres d'inventaire: "Coltello lungo 0.44, largo 0.045 appuntito, mentre che il manico, mai coperto di legno o d'altro, si allunga in una coda lunga 0.14 ". Voir également $N S c$ 1913, p. 61. 


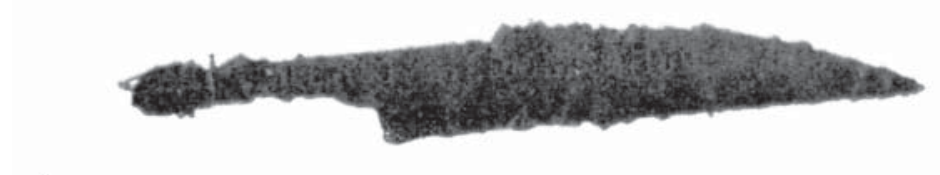

a)

b)

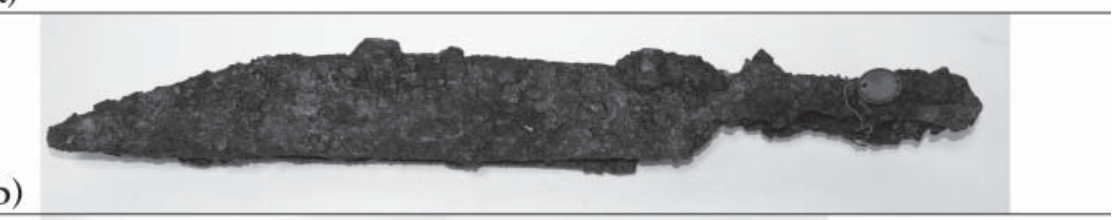

c)

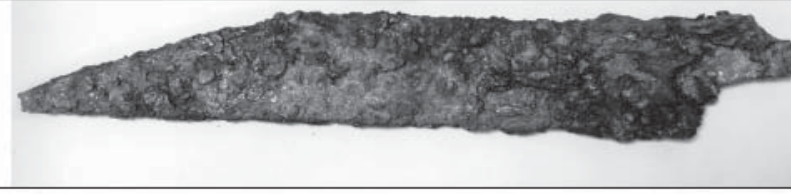

d)
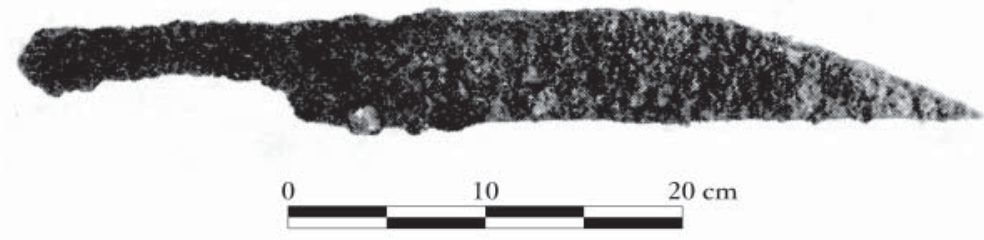

Fig. 16. Couteaux longs de Pompéi (échelle 1/5). a) Une des sept lames découvertes en I 9, 2, associées à six couperets (Inv. 1705, détruit ; Notizie degli scavi di Antichità, 1913, fig. 6 p. 61) ; b) Quatre lames associées par l'oxydation, découvertes en I 7, 2 (Inv. 3076 ; photo N. Monteix) ; c) Deux lames associées par l'oxydation, découvertes en I 7, 2 (Inv. 3077 ; photo N. Monteix) ; d) Une des deux lames découvertes associées à un couperet en I 10, 8 (Inv. 5168, détruit ; Notizie degli scavi di Antichità, 1934, fig. 28 p. 318).

I 9, 2. ${ }^{41}$ [188] Il convient en revanche de rester prudent face à ce type d'assemblage de couteaux qui peuvent également avoir été employés dans des contextes domestiques de préparation culinaire. Ainsi, dans un espace de stockage - ou une cuisine temporaire de la maison I 10, 8, deux longs couteaux ont été découverts en association avec un couperet (fig. 14, c et $16, d){ }^{42}$

41 Six lames découvertes le 4 février 1923 dans l'angle nordouest de l'atrium de la maison I 7, 1, inventoriées sous les numéros 3076 (quatre lames associées par l'oxydation), 3077 (deux lames). Les deux possibles couperets, inventoriés sous le numéro 3078 ont été détruits par le bombardement. La description qui en est donnée par les livres d'inventaire permettrait de les identifier comme des couperets à lame arrondie : "Altre due lame di coltella, molto larghe alla punta, a taglio curvo, e rastremantesi verso il manico, sul quale ci sono delle strisce ornamentali di bronzo. Riunite insieme dall'ossido e prive dei rispettivi manici. Lungh. m. 0.225 Largh. 0.13, inferiore 0.04".

42 Découverts groupés le 28 novembre 1932 dans la pièce 8 de la maison I 10, 8, les deux couteaux longs ont été inventoriés
Un second groupe, en nombre beaucoup plus réduit, paraît nécessairement devoir être lié aux activités de boucherie. Il s'agit de deux couteaux provenant des commerces alimentaires $\mathrm{V} 4$, 6-7 et IX 11, 2 (fig. 17, a-b). ${ }^{43}$ Tous les deux se présentent sous la forme d'une lame en demie feuille de laurier, à soie plate. Le dos est légèrement arrondi, le tranchant droit et peu incliné par rapport au manche.

sous le numéro 5168 (l'un mesure $49 \mathrm{~cm}$ de long, l'autre $44 \mathrm{~cm}$ ), tandis que le couperet portait le numéro 5169. Détruits durant le bombardement de 1943, ils ne sont plus visibles que sur les photos publiées dans les NSc en 1934 (fig. 28 p. 319). Sur le caractère temporaire du stockage dans la pièce de découverte, dans laquelle de nombreux objets se rapportent aux activités culinaires, voir Penelope M. ALLISON, The house of the Menander at Pompeii. Vol. III : the finds, a contextual study (Oxford, 2006), pp. 353-354.

43 Couteau découvert le 23 octobre 1899 dans le commerce alimentaire V 4, 6-7. Inventorié sous le numéro 54281 (ex 1959). Couteau découvert le 16 janvier 1912 dans le commerce alimentaire IX 11, 2, dit 'd'Asellina'. Inventorié sous le numéro 1191. 


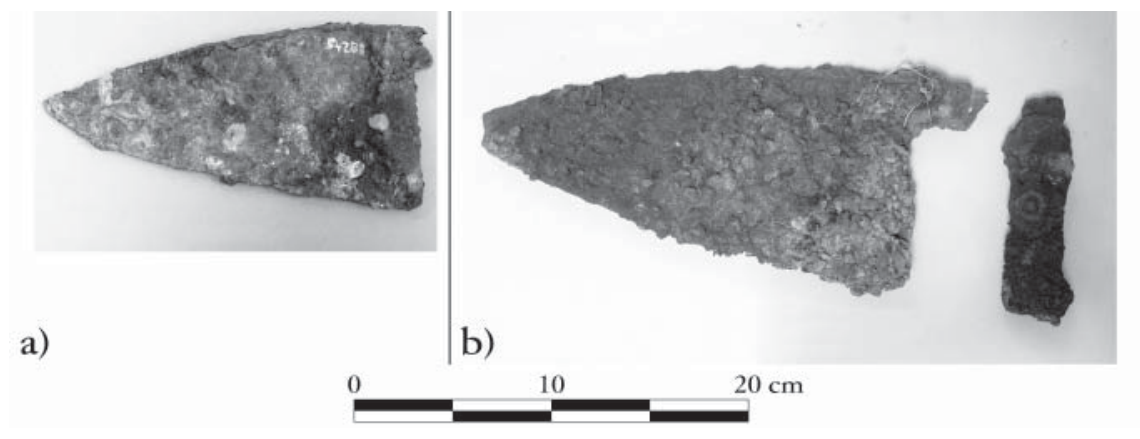

Fig. 17. Feuilles provenant de commerces alimentaires pompéiens (échelle 1/5). a) Couteau découvert en V 4, 6-7 (Inv. 54281 [ex 1959]) ; b) Couteau découvert dans le commerce alimentaire "d’Asellina” (IX 11, 2 ; Inv. 1191) (photos N. Monteix).

a)

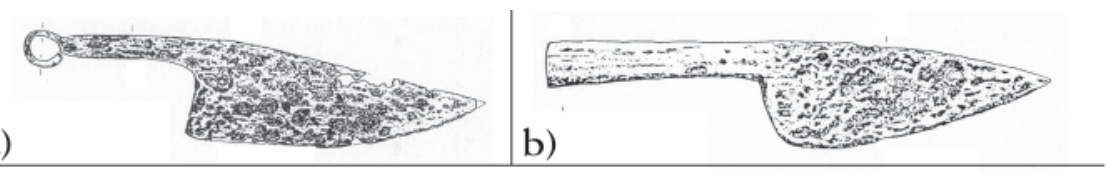

c)
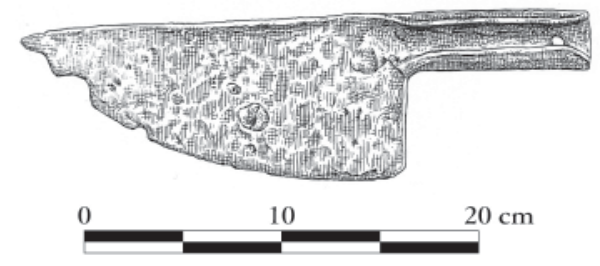

Fig. 18. Feuille et couteaux (échelle 1/5). a) et b) Couteaux de Sierentz (Christian VALLET, "Les outils de la découpe des viandes à Sierentz", Cahiers de l'Association pour la promotion de la recherche archéologique en Alsace, 11, 1995, fig. 7-8 p. 80-81) ; b) Feuille de River Churn, Cricklade, Wiltshire (British Museum ; W. H. MANNING, Catalogue..., pl. 57).

Ce dernier est fixé par enserrement du talon : une même feuille entoure l'arrière de la lame et se termine en manche. Cette technique de fixation est perceptible par le léger rehaussement du talon, malgré l'effet de lissage provoqué par la corrosion. Rappelons qu'elle est très distinctement représentée sur le relief [189] d'Aquilée (Fig. 2). Le contexte de découverte, deux commerces alimentaires, cauponae, ayant l'un comme l'autre des fonctions de restauration, l'un 'sur le pouce', l'autre avec une véritable salle d'accueil des hôtes, lie de façon très nette ces deux couteaux au découpage de la viande. Contrairement aux couperets que nous venons de voir, cette forme de lame est d'une part plus fréquente hors de Pompéi - des exemples morphologiquement proches, sauf pour la technique d'emmanchement, sont attestés aussi bien dans les collections du British Museum qu'à Sierentz (fig. 18, a-c) - , mais aussi plus fonctionnelle : la faible inclinaison de la lame par rapport au manche lui permet de servir de couperet, tandis que sa pointe et son poids moyen le rendent maniable pour des découpes plus précises. ${ }^{44}$ Il s'agit du couteau de boucher par excellence, la feuille, encore utilisée de nos jours. Si le contexte de découverte de ces deux couteaux est une indication claire pour percevoir une partie du marché de la viande dans les restaurants et commerces alimentaires, il faudrait rester prudent avant de considérer ces derniers comme l'unique lieu de découpe. Le dépouillement des inventaires a ainsi permis de noter des découvertes de gros couteaux isolés dans

44 W. H. MANNING, Catalogue..., cat. Q 96, Q 98-100 p. 122 et pl. 57; Ch. VALLET, “Les outils de la découpe...", p. 79-81. 


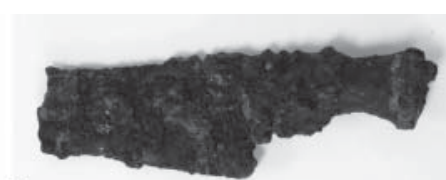

a)
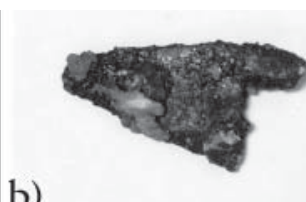

b)
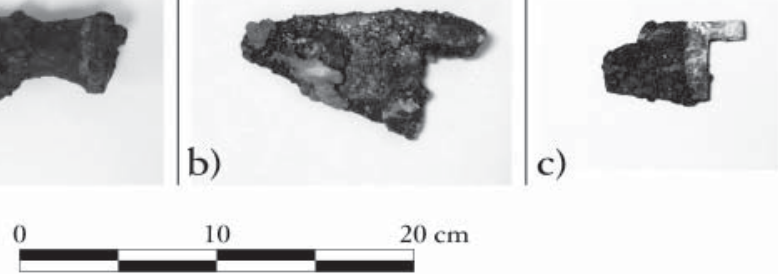

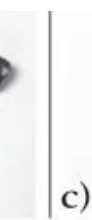

$20 \mathrm{~cm}$

Fig. 19. Couteaux à lame triangulaire de Pompéi (échelle 1/5). a) Couteau provenant de l'étage d'une maison dans

l'îlot V 4 (Inv. 2331) ; b) Couteau découvert sous un escalier dans la maison I 9, 10 (Inv. 9762) ; c) Couteau provenant éventuellement de la villa 'de la Pisanella' à Boscoreale (Inv. 18189) (photos N. Monteix).

des espaces strictement domestiques qui ne sont pas pour autant des cuisines. ${ }^{45}[190]$

Enfin, d'autres couteaux pourraient être considérés comme liés à la découpe de la viande. Il s’agit de trois exemplaires provenant tous de contextes domestiques - un appartement à l'étage, un rangement sous un escalier et peut-être la Villa de la Pisanella à Boscoreale (fig. 19, a-c). ${ }^{46}$ Tous les trois sont courts, moins d'une dizaine de centimètres, présentent une lame triangulaire dont l'angle empêche qu'ils aient été utilisés comme couperet, même pour des os fins, sauf à songer à une taille en percussion semi-posée. La forme de la lame est par trop fréquente sur les représentations de couteaux peu détaillées pour que l'on ne les considère pas comme destinés à couper de la chair, dans un contexte domestique.

\section{Conclusions}

En dépit du caractère éminemment préliminaire de ces remarques, quelques conclusions peuvent être tirées de cette première approche des couteaux de Pompéi. Tout d'abord, le couperet n'est pas le seul instrument utilisé par les bouchers. L'ensemble des sources l'indique, qu'elles soient archéozoologiques,

45 Un 'grosso coltello' a été mis au jour le 27 février 1877 dans la pièce $\mathrm{f}$ de la maison VI 14, 38, flanquant le nord de l'atrium (NSc 1877, p. 63). Avant d'interpréter cette découverte dans une pièce qui n'est pas une cuisine - type d'espace dont semble être dépourvue cette maison -, il serait impératif de pouvoir observer directement le couteau s'il existe encore.

46 Couteau à lame triangulaire en fer et manche recouvert de bois, découvert le 14 janvier 1901 à l'étage d'une maison située dans l'îlot V 4, inventorié sous le numéro 2331 ; couteau à lame triangulaire, fragmentaire, découvert le 13 août 1952 sous un escalier dans la maison I 9, 10, inventorié sous le numéro 9762 ; couteau à lame triangulaire en fer (fragmentaire), emmanché de iconographiques ou matérielles. L'influence forte des reliefs figurant les activités de boucherie m'a amené à essentiellement considérer les lames triangulaires comme devant être associées aux pratiques de boucherie, entendue au sens le plus large de découpage de la viande. Quatre types de couteaux ont été mis en évidence dans ce cadre [191], le couperet, le long couteau à dresser des filets ou à inciser, la feuille tout autant couperet que couteau de découpe - et enfin le petit couteau triangulaire. ${ }^{47}$ En l'état actuel du dépouillement des archives, ce dernier apparaît exclusivement dans des contextes domestiques. En revanche, les trois autres types ont été mis au jour selon des faciès remarquables, liés aux fonctions que l'on peut leur accorder : les couperets sont fréquemment associés aux longs couteaux utilisés pour tailler des filets, tandis que les feuilles sont toujours seules, en raison de leur fonction double de débitage des os et de découpe de la viande. Notons que si la découverte d'un ou plusieurs de ces instruments tranchants permet de caractériser [192] une activité de boucherie, elle n'indique pas nécessairement la présence d'un boucher - au sens d'artisan spécialisé dans la transformation d'une carcasse en pièces de viande consommables.

bronze, provenant éventuellement de la villa 'de la Pisanella' à Boscoreale, inventorié sous le numéro 18189.

47 Cette première typologie donne des résultats plus fins que la seule analyse textuelle, qui mentionne tout au mieux deux termes pour les couteaux utilisés en boucherie : culter et dolabra (Paul., 2 ad vitell. [Dig. 33, 7, 18 pr.]). Il semble préférable d'éviter d'employer des termes latins pour caractériser ces instruments, au même titre que limiter l'utilisation de culter au sens de couteau de sacrifice est par trop restrictif et inexact (contra Anne Viola SIEBERT, Instrumenta sacra. Untersuchungen zu römischen Opfer-, Kult- und Priestergeräten, Religionsgeschichtliche Versuche und Vorarbeiten 44 (Berlin/New York, 1999), pp. 79-84). 

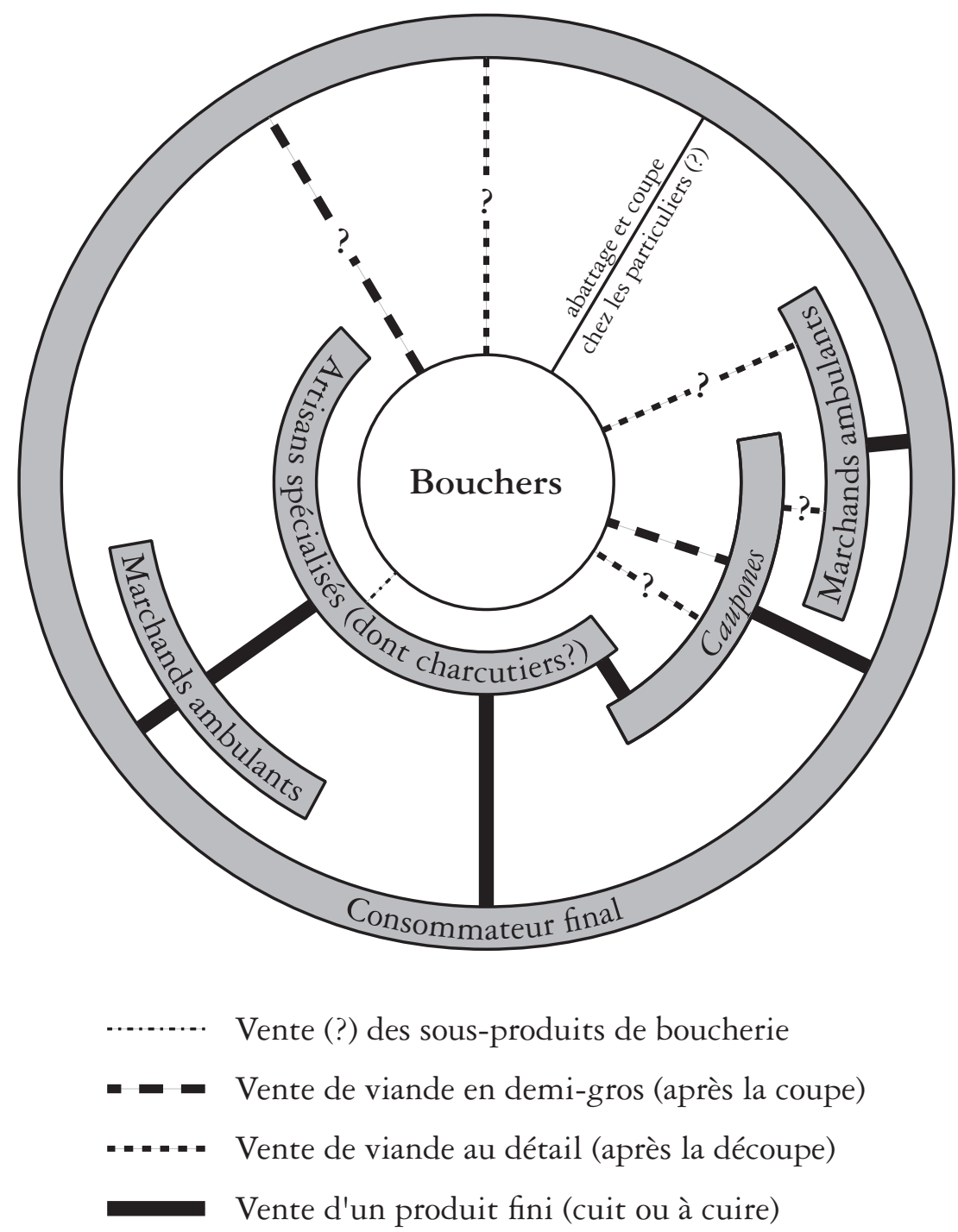

Fig. 20. Proposition de schéma d'organisation du marché de la viande à Pompéi (N. Monteix).

Par-delà cette remarque de prudence et en rassemblant une documentation éparse et encore très lacunaire, il est possible de proposer un schéma - hypothétique - d'organisation du marché de la viande à Pompéi (fig. 20).

Les dix couperets découverts en deux points de la ville semblent indiquer que les premières phases de la boucherie et particulièrement la coupe - si ce n'est également l'éviscération et les autres opérations préalables comme l'abattage - pourraient avoir été effectuées hors du point de découverte des couteaux. Les bouchers de Pompéi pourraient ainsi n'avoir pas été liés à un espace particulier. Ils se déplaceraient vers la viande à couper au lieu que celle-ci leur soit apportée. ${ }^{48}$ Seul le macellum pourrait prétendre à la concentration des activités de coupe, très certainement en lien avec la concentration de 'matière première' induite par la proximité des grands sanctuaires de la ville comme le rappelle W. van Andringa dans le présent volume. Quant aux quantités vendues dans cet édifice public, nous en sommes réduits aux supputations : aucun élément ne permet en l'état actuel de définir avec certitude si les 'coupeurs' rassemblés au macellum, préparateurs de viande en

48 La présence de bouchers lors des sacrifices pour préparer la carcasse une fois qu'elle a perdu son caractère sacré n'est pas à exclure. 
demi-gros, pratiquaient également la découpe des quartiers en morceaux de taille plus réduite. Il est probable que c'est au moment de la coupe - juste avant celle-ci - qu'intervenaient différents artisans pour se procurer les sous-produits de la boucherie (sang, toison, tripes, suint, cornes et os), matière première nécessaire à l'exercice de leur métier.

Un dernier point concernant les premières opérations bouchères doit être souligné : la découverte dans des espaces domestiques d'instruments permettant la coupe et la découpe - couperets et longs couteaux - laisse toutefois supposer que les techniques de boucherie pouvaient ne pas être mises en pratique par des hommes de l'art mais par le personnel attaché à la maison.

Une fois la carcasse débitée, des morceaux de taille conséquente sont achetés par les caupones, tenanciers des commerces alimentaires répartis à travers la ville, comme cela peut se déduire sans grand risque d'erreur grâce aux feuilles retrouvées dans leurs locaux commerciaux, trop petits pour per- mettre l'abattage, même d'un mouton. En revanche, le lieu d'approvisionnement du marchand ambulant identifié dans la Palestre est délicat à déterminer. Il peut s'agir tant du macellum, si la vente au détail s'y déroulait, que directement chez les caupones [193].

Le consommateur final, qu'il ait mangé de la viande directement dans les commerces alimentaires, qu'il l'ait achetée chez ces même restaurateurs ou sur le marché, voire qu'il l'ait abattue chez lui, semble avoir eu la possibilité de se fournir en viande dans plusieurs points de la ville. Toutefois, plusieurs questions restent en suspens: où travaillaient les charcutiers dont les graffitis nous vantent les produits ? Quels intermédiaires, si tant est qu'ils aient existé, se trouvaient entre les bouchers découpant la carcasse et les divers revendeurs ? La part de la viande dans l'alimentation des Pompéiens était-elle aussi faible que ce que les graffites laissent paraître ? La poursuite du dépouillement des archives de fouilles permettra peut-être de répondre à une partie de ces questions. 


\section{Annexe : la viande dans les graffites de Pompéi}

CIL IV, 5380

Inscription lue sur le mur de la pièce $t$ de la maison IX 7, 25, dont une grande partie pourrait avoir des fonctions de restauration ou d'hôtellerie.

\begin{tabular}{|c|c|c|c|}
\hline \multirow[t]{4}{*}{ VIII Idus } & Casium I (asse) & servato & $\begin{array}{l}\text { Idubus (sic) } \\
\text { Pane(m) II (assibus) } \\
\text { Pane(m) cibar(ium) II (assibus) }\end{array}$ \\
\hline & Pane(m) VII (assibus) & Montana (denario) I & Oleum V (assibus) \\
\hline & Oleum III (assibus) & Oleum (denario) I VIIII (assibus) & Halica(m) III (assibus) \\
\hline & Vinum III (assibus) & $\begin{array}{l}\text { Pane(m) IV (assibus) } \\
\text { Casium IV (assibus) }\end{array}$ & $\begin{array}{l}\text { Domat[ori] } \\
\text { Pisciculum II }\end{array}$ \\
\hline \multirow[t]{5}{*}{ VII Idus } & Pane(m) VIII (assibus) & $\begin{array}{l}\text { Porrum I (asse) } \\
\text { Por(rum) patella(m) I (asse) }\end{array}$ & \\
\hline & Oleum V (assibus) & $\begin{array}{l}\text { Sittuae VIIII (assibus) } \\
\text { Inltynium I (asse) }\end{array}$ & \\
\hline & Cepas V (assibus) & & \\
\hline & Pultarium I (asse) & III Idus Pane(m) II (assibus) & \\
\hline & Pane puero II (assibus) & Pane(m) puero II (assibus) & \\
\hline \multirow[t]{3}{*}{ VI Idus } & $\begin{array}{l}\text { Vinum II (assibus) [194] } \\
\text { Pane VIII (assibus) }\end{array}$ & \multicolumn{2}{|l|}{$\begin{array}{l}\text { Pri(die) Idus Puero pane II (assibus) } \\
\text { Pane(m) Cibar(ium) II (assibus) }\end{array}$} \\
\hline & Puero pane(m) IV (assibus) & Porrum I (asse) & \\
\hline & Halica(m) III (assibus) & & \\
\hline V Idus & $\begin{array}{l}\text { Vinum domatori (denario) } \\
\text { Pane(m) VIII (assibus) vinuı }\end{array}$ & sibus) casium II (assibus) & \\
\hline IV Idus & $\begin{array}{l}\text { Hxeres (denario) } \\
\text { Pane II (assibus) } \\
\text { Femininum VIII (assibus) } \\
\text { Triticum (denario) I } \\
\text { Bubella(m) (pro bubulam) } \\
\text { Palmas I (asse) } \\
\text { Thus I (asse) } \\
\text { Casium II (assibus) } \\
\text { Botellum I (asse) } \\
\text { Casium molle IV (assibus) } \\
\text { Oleum VII (assibus) }\end{array}$ & & \\
\hline
\end{tabular}

CIL IV, 8561

Inscription lue sur la colonne XVIII de la Palestre (portique méridional).

Pompe(iis) iu(?) a(ssibus) III S(emis)

$\mathrm{P}$ (ondo ?) lard(i) a(ssibus) III

Vinum a(sse) S(emis)

Casium a(sse) S(emis)

Oleum a(sse) I

Panem a(ssibus) II

Suar(ium?) a(ssibus) IIII [195] 
CIL IV, 8566 b

Inscription lue sur la colonne XVIII de la Palestre (portique méridional)

Pane(m) a(ssibus) II

Purm(entarium) a(ssibus) III

oleum a(sse) I

[mot incompréhensible] a(ssibus) IIII

vinum a(sse) s(emis)

casium a(sse) s(emis)

[mot incompréhensible] a(ssibus) IIII [?]

vinum a(sse) s(emis)

XI K(alendas) ac(ce)pi (denarium) I

vinum a(sse) s(emis)

porcin(um) a(sse)

vinum a(sse) s(emis)

casium a(sse) s(emis)

L(uci) Gavi (asses) IIII s(emis) [accepi?]

vinum a(sse) s(emis)

$\operatorname{carn}(\mathbf{e m})$ a(sse) I

vinum a(sse) I

Si ce graffite a été interprété comme une liste de courses, la relecture 'asses' d'un signe illisible à la ligne 14 - à la place du 'denarios' interprété par M. Della Corte - permet d'y voir un compte attendant d'être soldé. Un vendeur ambulant - qui ne disposait pas d'un local construit et permanent - de denrées alimentaires aurait fait crédit à son client, jusqu'au 11e jour avant les calendes d'un mois et d'une année indéterminés, date à laquelle il touche 1 denier (16 as) pour une dette de 15 as et demi. Plus tard, il reçoit 4 as et demi d'un certain L. Gavius, qui ne doit pas nécessairement être identifié avec le client, alors que seuls 2 as sont dus. La liste s'achève avec la régularisation des comptes et une égalité des dépenses et des recettes à hauteur de 20 as et demi [196]. 